\title{
Experimental Results for Information Systems Based on Accesses Locality via Intuitionistic Fuzzy Metrics
}

\author{
Francisco Castro-Company ${ }^{1}$ and Salvador Romaguera, ${ }^{*}$ \\ ${ }^{1}$ Universidad Politécnica de Valencia, Spain \\ ${ }^{2}$ Instituto Universitario de Matemática, Pura y Aplicada, IUMPA-UPV, Universidad Politécnica de Valencia, 46071 \\ Valencia, Spain
}

\begin{abstract}
The notion of an intuitionistic fuzzy metric space is a natural generalization of the fuzzy metric space concept which provides mechanisms to measure the degree of nearness and remoteness between two elements of a fuzzy set according to a parameter $t$. In this work we show how, interpreting t as a value representing the evolution time of an information system, we can use effective prediction tools in systems that show a strong locality component and where operations require the coordination of actions over the set of elements.

Furthermore we show how we can tune the fuzzy metric results in order to predict access histories working on variations of the fuzzy constructions. To this end we study the suitability of a set of continuous t-norms and t-conorms to build fuzzy constructions. We have evaluated the metrics suitability according to their computation time and to the sensitiveness for different representative cases.
\end{abstract}

\section{INTRODUCTION}

Since the theory of fuzzy sets introduced by L.A. Zadeh [1] appeared in 1965 it has been used in a range of areas of mathematics. One of these areas, fuzzy logic, has allowed to apply fuzzy behaviour to implement industrial control devices and to use multivalued logic concepts to real scenarios.

A fuzzy set is a set whose elements may be divided into the ones that belong to the set, the ones that do not belong to the set and the ones for which it is not possible to decide without a certain degree of uncertainty whether they belong to the set or not. Following Zadeh's idea, K. Atanassov [2] introduced the concept of intuitionistic fuzzy set to allow grouping elements according to degrees of nearness and remoteness.

Fuzzy topology is another example of use of Zadeh's theory. Authors of this field have pursued the definition of a fuzzy metric space from different points of view (see [3-6], etc.).

This work deals with the use of fuzzy metric concepts to achieve accesses optimization in information systems in general. Among the variety of information systems, we choose those based on access locality in the sense of nearness among the elements of the set. This characteristic appears quite often in basic information systems (compilation, physical memory accesses, transaction isolation, etc.) and also suits finely the way human organizations are structured (headquarters and geographically scattered delegations for instance).

*Address correspondence to this author at the Instituto Universitario de Matemática Pura y Aplicada IUMPA-UPV, Universidad Politécnica de Valencia.46071 Valencia, Spain; E-mail: sromague@mat.upv.es
After a brief review to the results presented in the starting point section using a quasi-metric lattice, we will show that the Kramosil-Michalek definition of fuzzy metric [3] is the one that is better suited for our purposes. The metric formed by the chosen set and a distance (t-norm) on the elements of the set allows to use fuzzy metric and intuitionistic fuzzy metric techniques to improve our previous results.

As a core introduction for our work we will display a series of measurements with experiments using several continuous t-norms and t-conorms that allow us to construct the fuzzy metric (and the intuitionistic fuzzy metric) in a way that its results approximate to different real systems behaviours.

\section{STARTING POINT}

In [7] we tackled the problem of detecting data access patterns with several degrees of locality using a quasi-metric lattice. The quasi-metric lattice is based upon a quasi-metric space $(X, d)$ where $X$ is the non-empty set of objects of the system and $d$ is a quasi-metric on $X$.

Using the quasi-metric space and the induced order $x \leq_{d} y \Leftrightarrow d(x, y)=0$ on the set $X$, we obtained an ordered set $\left(X, \leq_{d}\right)$ that allowed us to build a quasi-metric lattice.

\section{Definition 1 (Quasi-Metric Lattice)}

A quasi-metric lattice is a triple $(X, d, \leq)$ such that $(X, d)$ is a quasi-metric space and $(X, \leq)$ is a lattice such that for $x, y, z \in X$ :

$$
d(x \vee z, y \vee z) \leq d(x, y) \text { and } d(x \wedge z, y \wedge z) \leq d(x, y)
$$

For each $x \in X$, denote by $k(x)$ the number of uses of $x$ in $[0, T]$, where $T$ is the instant of time when we want to pre- 
dict $x$ 's value reliability and $x$ is an object in an information system such as a replicated database object for example (see [7]).

Now, for each $x \in X$ with $k(x)>0$, we construct a function also denoted by $x$ from $[0, T]$ into $\mathbf{N} \cup\{0\}$ as follows:

$$
\begin{aligned}
& x(t)=0, \text { if } t=0 ; \\
& x(t)=1, \text { if } 0<t \leq t_{1(x)} ; \\
& x(t)=2, \text { if } t_{1(x)}<t \leq t_{2(x)} ;
\end{aligned}
$$

$x(t)=k(x)-1$, if $t_{(k(x)-2)(x)}<t \leq t_{(k(x)-1)(x)}$,

$x(t)=k(x)$, if $t_{(k(x)-1)(x)}<t \leq T$.

If $k(x)=0$, we define $x:[0, T] \rightarrow[0,1]$ by

$x(t)=0$, if $0 \leq t \leq T$.

$x(t)$ represents object $x$ history of accesses during time.

Next we are interested in obtaining a function $v$ from $X$ into $[0,1]$ such that $v(x)$ provides a sufficiently satisfactory value of the probability of "use" of $x$ and satisfying the following reasonable and obviously desirable fact to model locality:

\section{Proximity and Frequency Condition}

If $x, y \in X$ satisfy that $0<k(y) \leq k(x)$ and for each $j \in\{1, \ldots, k(y)\}$ there is $i \in\{1, \ldots, k(x)\}$ with $t_{j(y)} \leq t_{i(x)}$ then $v(y) \leq v(x)$; in addition, if for some $j \in\{1, \ldots, k(y)\}$ there is $i \in\{1, \ldots, k(x)\}$ with $t_{j(y)}<t_{i(x)}$ then $v(y)<v(x)$.

i.e., $v$ allows us to compare two elements histories in a way that if the second element history adds closer to $T$ accesses in between the first element history then $v$ value for the second element is greater than for the first object.

A relatively easy function which is a suitable candidate to provide an efficient model in our study, and whose construction is suggested by the function $x(t)$ given above, is the function $v: X \rightarrow[0,1]$ defined as follows:

$$
\text { If } k(x)=0 \text {, then }
$$

$$
v(x)=0,
$$

and if $k(x)>0$, then

$$
v(x)=\sum_{j=1}^{k(x)} 2^{-j} \frac{t_{(k(x)-(j-1))(x)}}{T} .
$$

In [7] it is shown how $v$ satisfies the nearness and frequency condition and how the quasi-metric lattice offers an adequate framework to explain the pattern accesses properties by grouping objects in classes $[x]=\{y \in X: v(x)=v(y)\}$ in a way that if we compare two classes $[x] \sqsubseteq[y]$ $\Leftrightarrow v(x) \leq v(y)$, then $\tilde{X}:=\{[x]: x \in X\}$ admits a lattice structure and $(\tilde{X}, d, \sqsubseteq)$ is a quasi-metric lattice.

\section{MODEL EXTENSION IN TIME: FUZZY METRICS}

As a natural continuation of the initial study we pretend to take advantage of the intermediate accesses values.
We will start the extension approach by recalling some basic definitions.

\section{Definition 2 (Metric)}

A metric on a set $X$ is a real valued function $d: X \times X \rightarrow \mathbf{R}$ such that for all $x, y, z \in X$ :

(i) $d(x, y) \geq 0$;

(ii) $d(x, y)=0 \Leftrightarrow x=y$;

(iii) $d(x, y)=d(y, x)$;

(iv) $d(x, y) \leq d(x, z)+d(y, z)$.

\section{Definition 3 (T-Norm [8])}

A continuous t-norm is a binary operation $*:[0,1] \times[0,1] \rightarrow[0,1]$ such that:

$*$ is commutative and associative, $*$ is continuous, $a * 1$ $=a$ for all $a \in[0,1]$ and $a * b \leq c * d$ when $a \leq c$ and $b \leq d(a, b, c, d \in[0,1])$

\section{Definition 4 (T-Conorm [8])}

A continuous t-conorm is a binary operation

$$
\diamond:[0,1] \times[0,1] \rightarrow[0,1] \text { such that: }
$$

$\diamond$ is commutative and associative, $\diamond$ is continuous, $a \diamond 0$ $=a$ for all $a \in[0,1]$ and $a \diamond b \leq c \diamond d$ when $a \leq c$ and $b \leq d(a, b, c, d \in[0,1])$.

\section{Definition 5 (Fuzzy Metric Space: Kramosil-Michalek [3])}

A triple $(X, M, *)$ is a fuzzy metric space if $X$ is an arbitrary set, $*$ is a continuous t-norm and $M$ is a fuzzy set on $X^{2} \times[0, \infty)$ such that (with $x, y, z \in X$ and $t, s>0$ ):

1) $M(x, y, 0)=0$.

2) $M(x, y, t)=1$ for all $t>0 \Leftrightarrow x=y$.

3) $M(x, y, t)=M(y, x, t)$.

4) $M(x, z, t) * M(z, y, s) \leq M(x, y, t+s)$.

5) $M(x, y\rfloor:,[0, \infty) \rightarrow[0,1]$ is left continuous.

\section{BASIS OF THE FUZZY METRIC EXTENSION}

While in our initial approach we choose $k(x)$ as the number of uses of an object $x$ between 0 and $T$ and the instant of time when the metric is calculated.

Now we choose $t$, where $0 \leq t<T$, and define $k(x, t)=$ $i(x)$ if $t \in\left(t_{i(x)-1}, t_{i(x)}\right]$ with $i(x) \leq k(x)$, and $k(x, t)=k(x)$ if $t \in\left[t_{k(x)}, T\right]$. (We take $\left.t_{0}=0\right)$.

Thus, $k(x, t)$ is a function of the computed accesses until the instant $t$.

Then, we define $v: X \times[0, \infty) \rightarrow[0,1]$ by:

$$
v(x, 0)=0 \text {, }
$$




$$
v(x, t)=\sum_{j=1}^{k(x, t)} 2^{-j} \frac{t_{(k(x, t)-(j-1))(x)}}{T} .
$$

if $0<t \leq T$, and $v(x, t)=1$ if $t>T$.

Let's suppose that $v(x, T)$ offers a "reasonable" value of probability of $x$ being accessed at instant $T$. Then we can compare $v(x, t)$ and $v(y, t)$ and if they show similar values then we can try to advance the prediction of $y$ 's class, which will most possibly be $[y]=[x]$.

This comparison is represented by the fuzzy metric space defined by the triple $(X, M, *)$ where $X$ is a non-empty set, $*$ a continuous t-norm and $M$ a fuzzy set in $X \times X \times(0, \infty)$ defined by:

$$
M(x, y, t)=v(x, t) * v(y, t)
$$

In [9] we show the following result, which establishes that $(M, *)$ is a fuzzy metric as defined by KramosilMichalek for an adequate $v$.

Proposition 1: Let $v: X \times[0, \infty) \rightarrow[0,1]$ be any function such that for each $x \in X \quad v(x$,$) is a left continuous non-$ decreasing function (i.e., $v(x, t) \leq v(x, s)$ if $t \leq s)$. Then $(X, M, *)$ is a fuzzy metric space where:

(a) $M(x, y, 0)=0$.

(b) $M(x, x, t)=1$ for each $t>0$ and for each $x \in X$.

(c) $M(x, y, t)=v(x, t) * v(y, t)$ if $x \neq y$ for each $t>0$.

Notice that our function $v$ defined at the beginning of the subsection satisfies Proposition 1, i.e., for each $x, v$ is left continuous and non-decreasing.

\section{EVALUATED T-NORMS}

We have considered different continuous t-norms (check Dubois-Prade [10]) and compared them according to their results with the fuzzy metric in $v(x, t)$ :

- Minimum: $\min (x, y):=\left\{\begin{array}{l}x \text { if } x \leq y \\ y \text { if } y<x\end{array}\right.$

- $\quad$ Product: $\prod(x, y):=x y$

- Lukasiewicz: $\mathrm{W}(x, y):=\max \{x+y-1,0\}$.

- T-norm families: different parameter values will be compared.

- Frank family:

$$
\begin{aligned}
& \log _{s}\left(1+\frac{\left(s^{x}-1\right)\left(s^{y}-1\right)}{s-1}\right) \\
& \text { where } s>0, s \neq 1
\end{aligned}
$$

- Hamacher family:

$$
\frac{x y}{\alpha+(1-\alpha)(x+y-x y)}
$$

where $\alpha \geq 0$.

- Sugeno-Weber family:

$$
\max \left\{\frac{x+y-1+\lambda x y}{1+\lambda}, 0\right\}
$$

where $\lambda \geq-1$.

- Schweizer-Sklar family:

$$
\left(\max \left\{x^{-p}+y^{-p}-1,0\right\}\right)^{1 / p} \text {. }
$$

- Yager family:

$\max \left\{1-\left((1-x)^{p}+(1-y)^{p}\right)^{1 / p}, 0\right\}$

where $p \in(0, \infty)$.

- Dombi family:

$$
\frac{1}{1+\left(\left(\frac{1-x}{x}\right)^{\lambda}+\left(\frac{1-y}{y}\right)^{\lambda}\right)^{1 / \lambda}}
$$

where $\lambda \in(0, \infty)$.

- Dubois-Prade family:

$$
\frac{x y}{\max (x, y, \gamma)} \text { where } \gamma \in[0,1] \text {. }
$$

Comparisons using continuous t-norm families allow us to tune the predictions precision (check related figures to see how the parameter affects the evaluation).

\section{COMPARISON OF RESULTS}

We evaluate $v$ in $[0, T]$ and we arbitrarily set $T=1000$ to allow the prediction to range from no uses to plenty of them. $t$ instants have been chosen uniformly scattered through the interval.

Our tests are based on comparisons of $\mathrm{v}$ values during $[0, T]$ for two different objects $x, y$ (fuzzy set elements) using continuous t-norms. These differences are achieved applying localized variations in the first object $x$ to obtain $y$. That is how we model element accesses with degrees of nearness as it happens in systems with strong locality components.

Computing the variation of $y$ is a simple implementation of the Proximity and Frequency condition. We have tried 3 kinds of variations as we display in the following subsections.

\section{Random Variations - Random}

Figs. (1-3) show $\mathrm{n}$ additional accesses to element $y$ are performed randomly through the interval $[0, T]$.

Fig. (2) shows $v(x, t)$ and $v(y, t)$ values: The final value for both experiments is high because accesses are performed throughout the end of the study for both elements. Fig. (3) shows the fuzzy metric results for the minimum, product and Lukasiewicz t-norms. Figs. (4-6) are examples of the results obtained for the t-norm families.

For the case of the Hamacher family, there is no abrupt change in the family behaviour when we introduce changes 
in the parameter $\alpha$ value. For close to 1 values, Hamacher family gets close to the product results because as the parameter is placed in at the denominator, $\alpha$ increments mean that $M$ values will decrease. As there is no upper bound for $\alpha$ this family allows us to get lower values than the ones obtained using the Lukasiewicz t-norm for the metric construction.

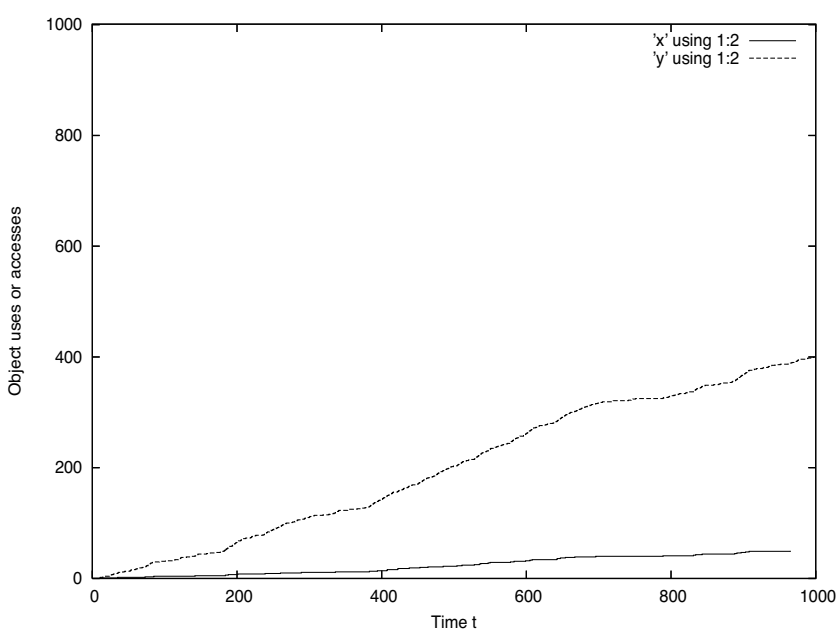

Fig. (1). Random histories.

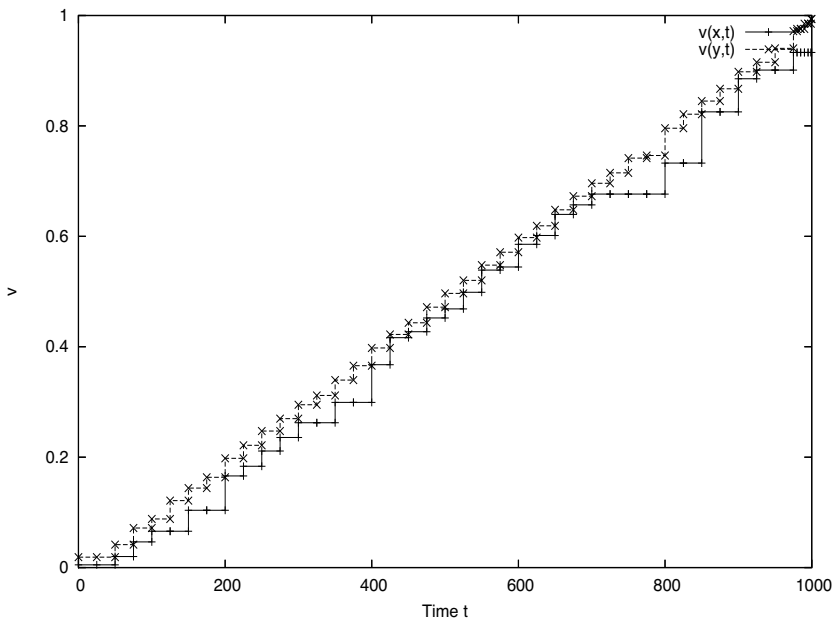

Fig. (2). Random: $v(x ; t)$ and $v(y ; t)$.

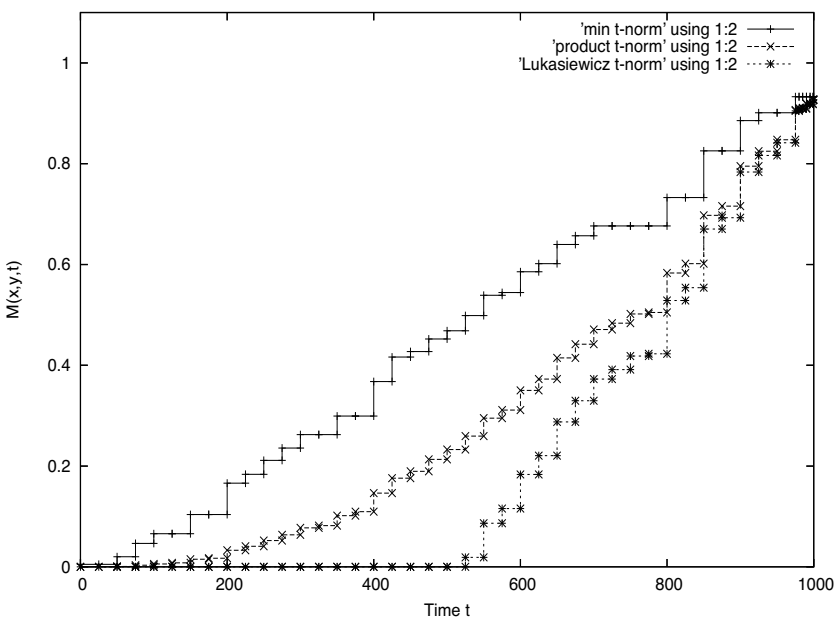

Fig. (3). Random: M using minimum, product, Lukasiewicz.

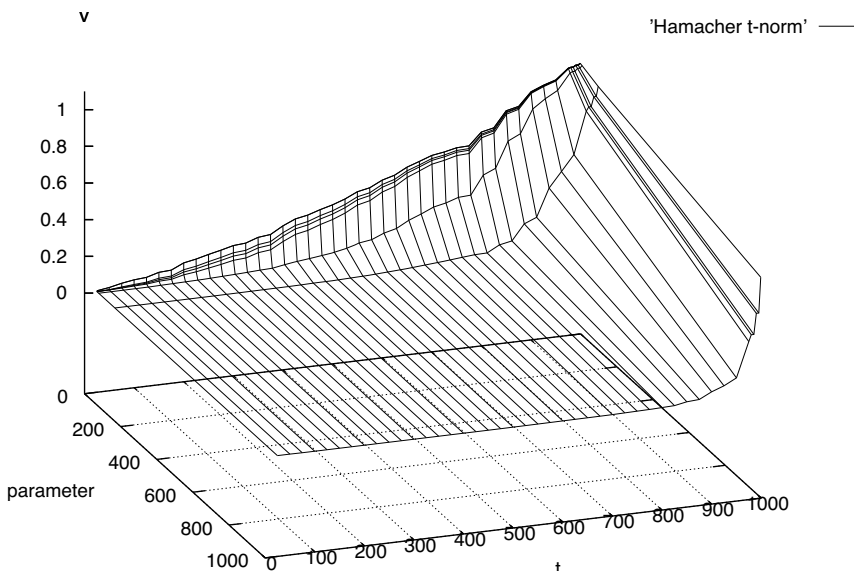

Fig. (4). Random: M using Hamacher.

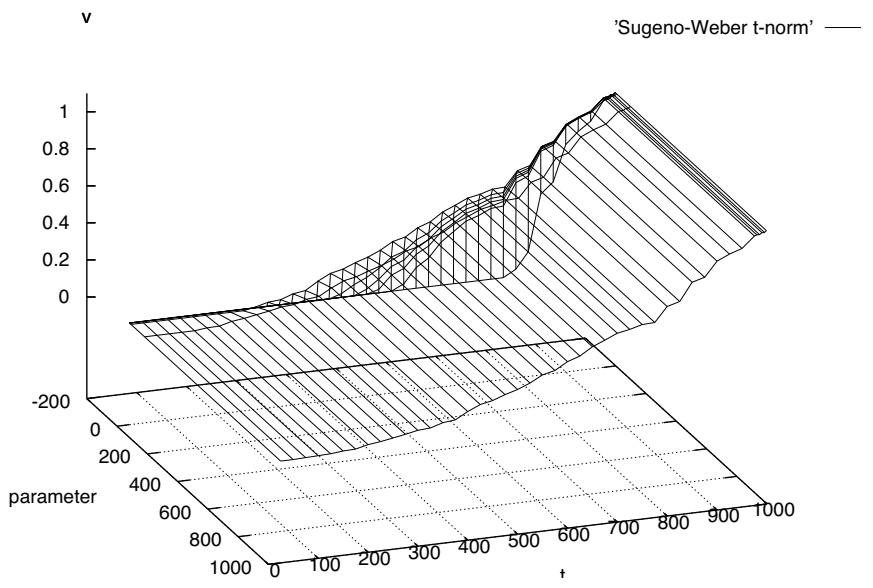

Fig. (5). Random: M using Sugeno-Weber.

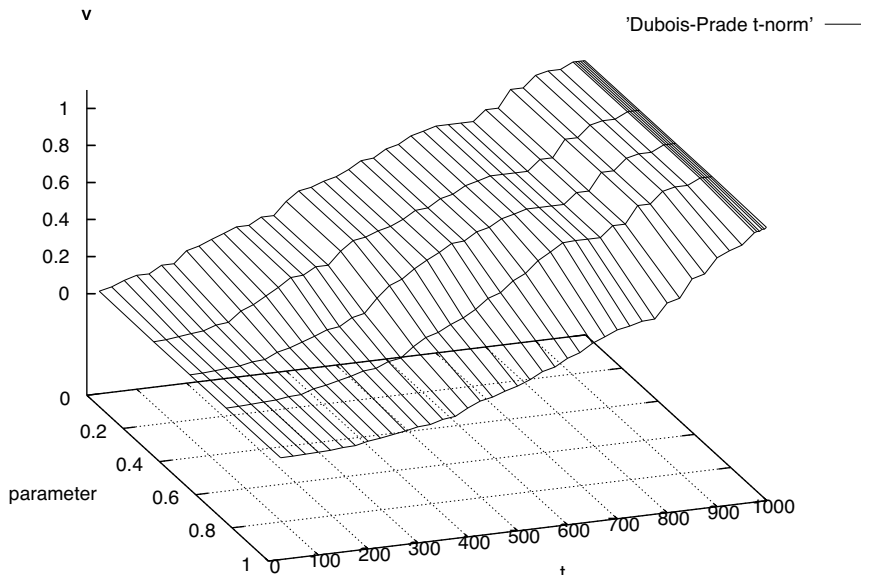

Fig. (6). Random: M using Dubois-Prade.

For the Sugeno-Weber t-norm with $\lambda$ values in between -1 and 0 the t-norm results converge to the Lukasiewicz ones and for $\lambda$ values greater than 0 results converge to the product ones.

For the Dubois-Prade results, increments imply a progressive decrement of $\mathrm{M}$.

\section{Left-Random Variations - Left-Random}

In this set of experiments, the $n$ additional accesses appear randomly close to the beginning of the history. It can be 
seen how the behaviour of the metric is similar to the one found in the previous case with completely random variations. This is due to the fact that early accesses have less impact on the final result and, in the end, the accesses distribution is quite similar to the random case.

See Figs. (7-9) for the data accesses distribution and $v$ and $\mathrm{M}$ evaluations. Figs. (10-12) are examples of the results obtained for $M$ built using three different t-norm families.

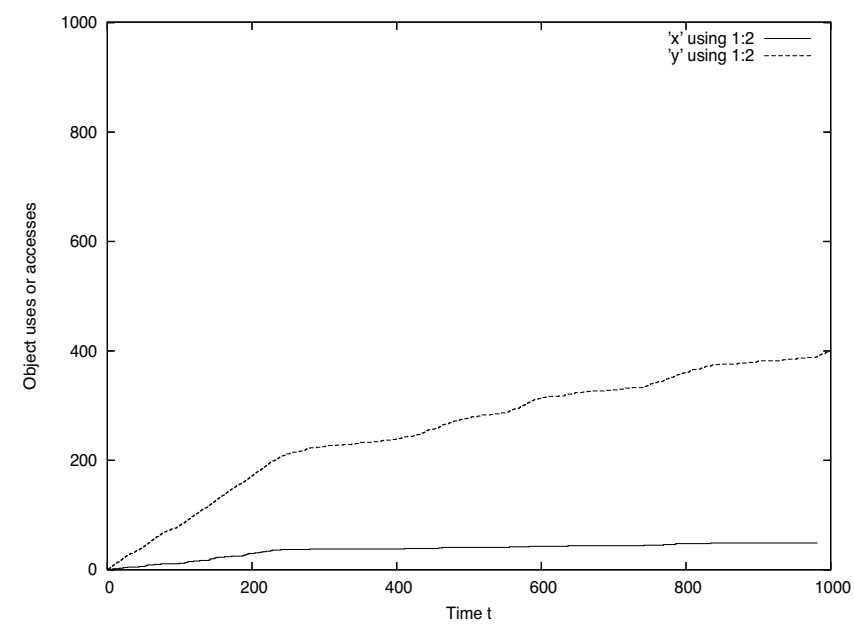

Fig. (7). Left-Random accesses.

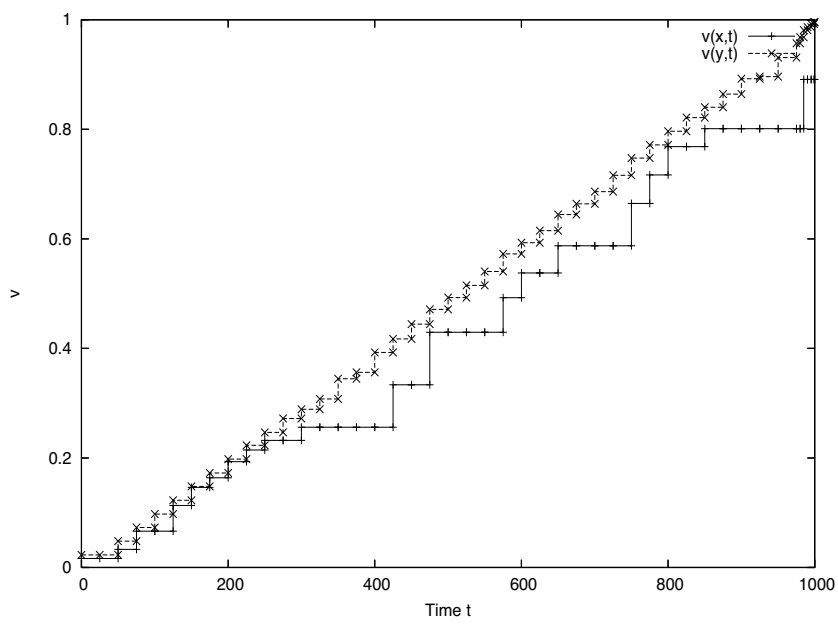

Fig. (8). Left-Random: $v(x ; t)$ and $v(y ; t)$.

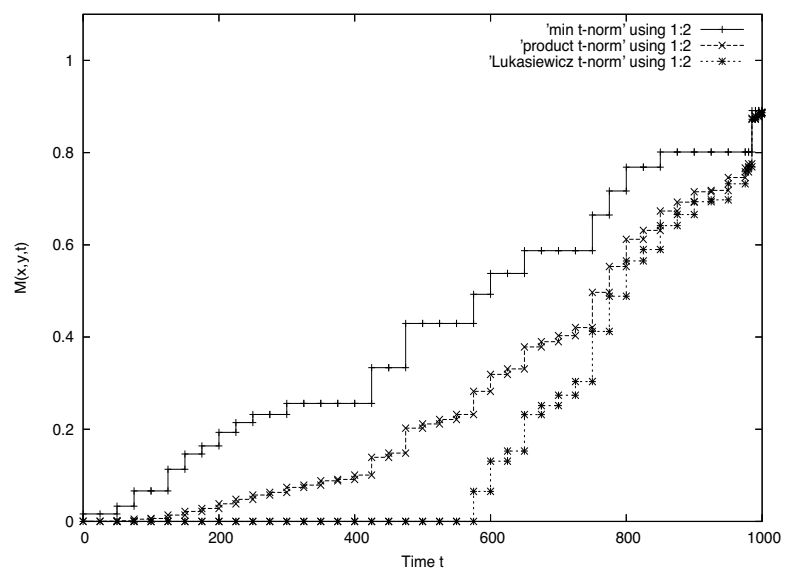

Fig. (9). Left-Random: $\mathrm{M}$ using minimum, product and $\mathrm{Lu}-$ kasiewicz.

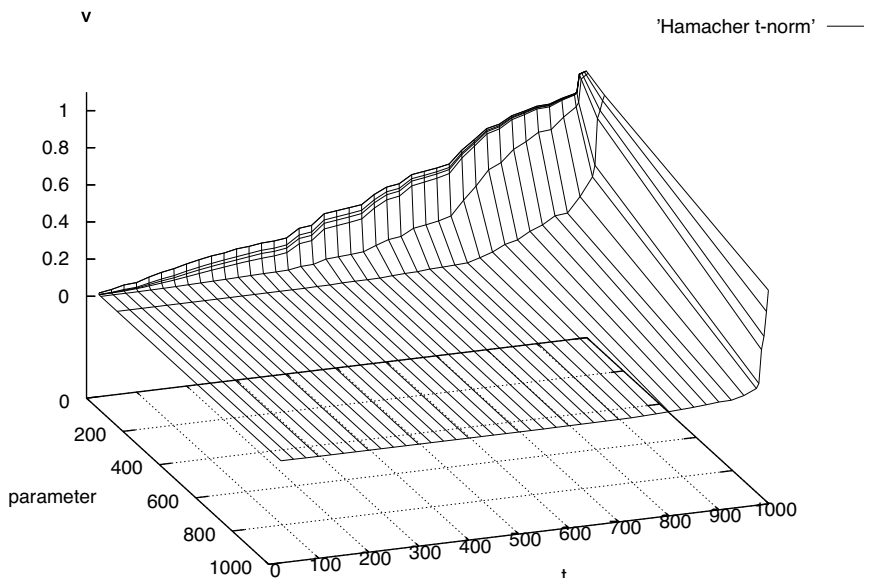

Fig. (10). Left-Random: M using Hamacher.

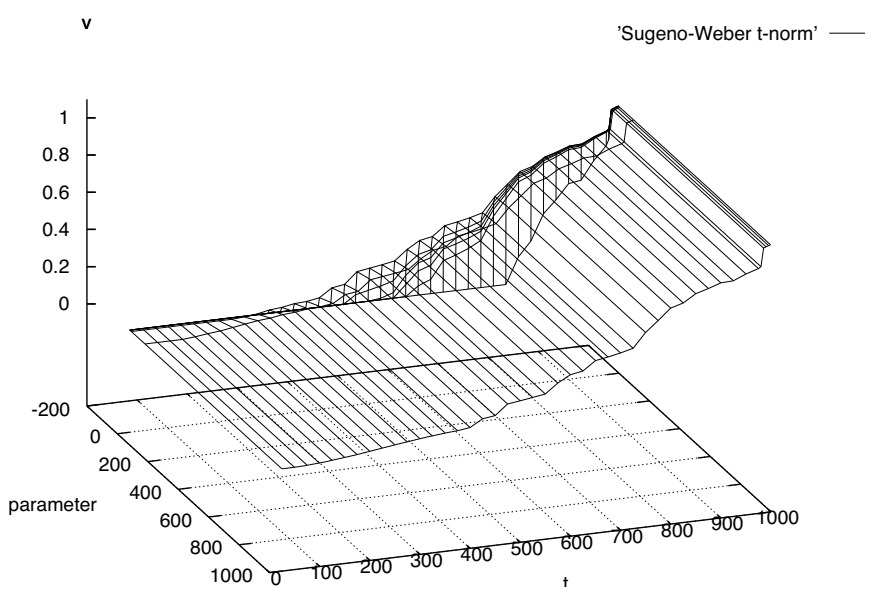

Fig. (11). Left-Random: M using Sugeno-Weber.

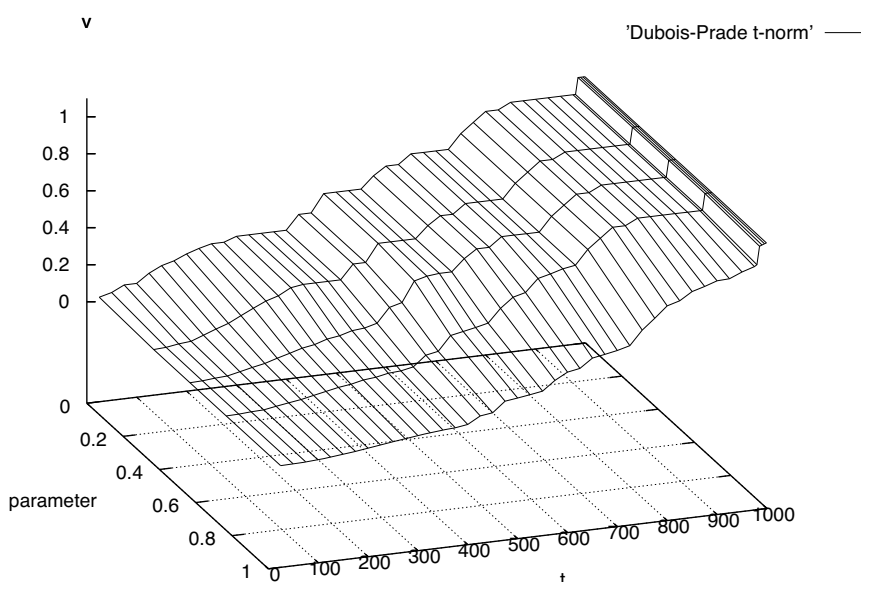

Fig. (12). Left-Random: M using Dubois-Prade.

\section{Right-Random Variations - Right-Random}

Now the $n$ additional accesses appear randomly close to the end of the history. As soon as the histories start diverging the metrics behaviour is similar to the previous experiments. In these experiments we can see how equal histories are perfectly identified as it happens simply with $\mathrm{v}$ evaluations but, as in all the experiments, $M$ evaluation allows a richer identification of differences.

See Figs. (13-15) for the distribution of accesses and Figs. (16-18) as representative examples of the results ob- 
tained for different fuzzy metrics built using different t-norm families.

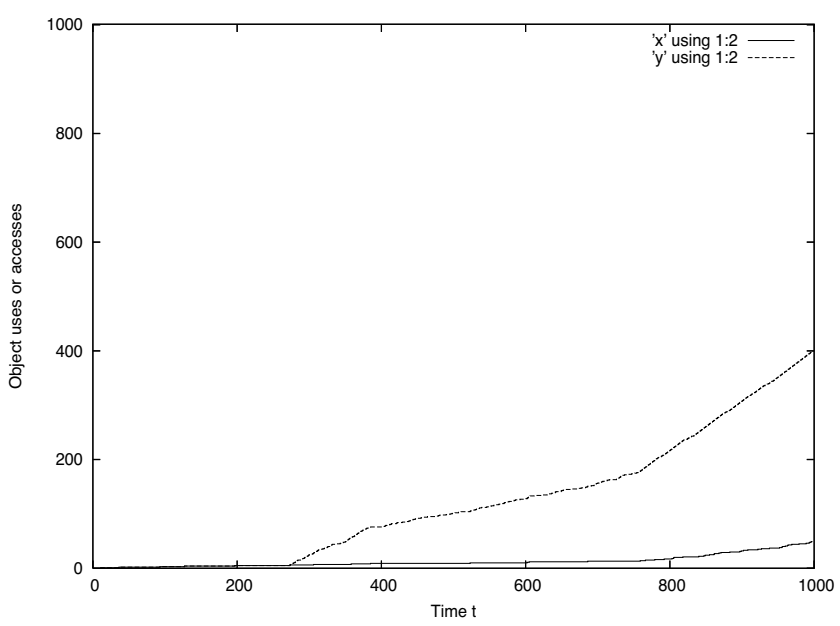

Fig. (13). Right random accesses.

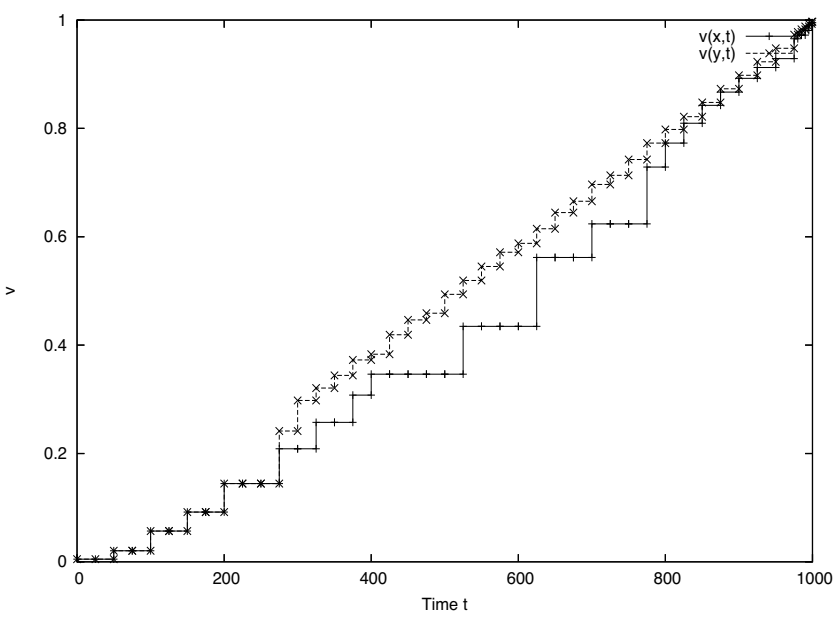

Fig. (14). Right random: $v(x ; t)$ and $v(y ; t)$.

\section{Two Independent Histories - Opposite Histories}

One of them has accesses performed mainly at the beginning and the other has them at the end. Experiments can be observed in Figs. (19-21). Figs. (22-24) are examples of the results obtained for the t-norm families.

In all previous experiment groups, final values for $M$ were big enough to consider that the element was likely to be accessed again soon. The reason for this constant behaviour is the fact that there were always close to $T$ accesses. The experiment with two independent histories shows a different case and $M$ evaluation does not seem to imply a prompt access happening. Even though the prediction is different, it can be noticed that the t-norms comparison is still valid.

\section{Additional t-Norms}

Next, we show in a separate group of figures families Schweizer-Sklar (Figs. 37-40), Dombi (Figs. 25-28), Frank (Figs. 29-32) or Yager (Figs. 33-36).

They require costly calculations which do not easily apply to our fast prediction intentions and neither of them introduces any characteristic which could not be found in the faster families or the traditional t-norms.

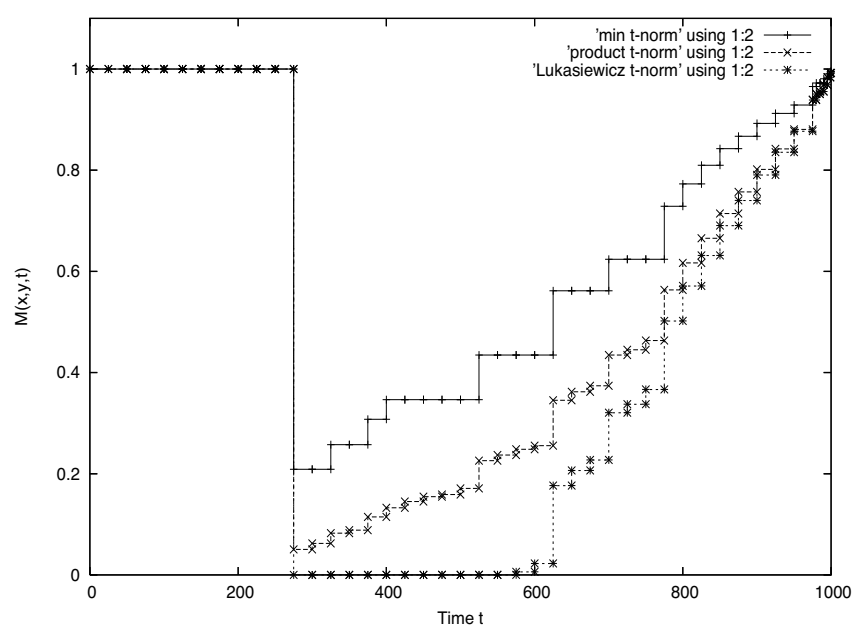

Fig. (15). Right random: $M$ using minimum, product, Lukasiewicz.

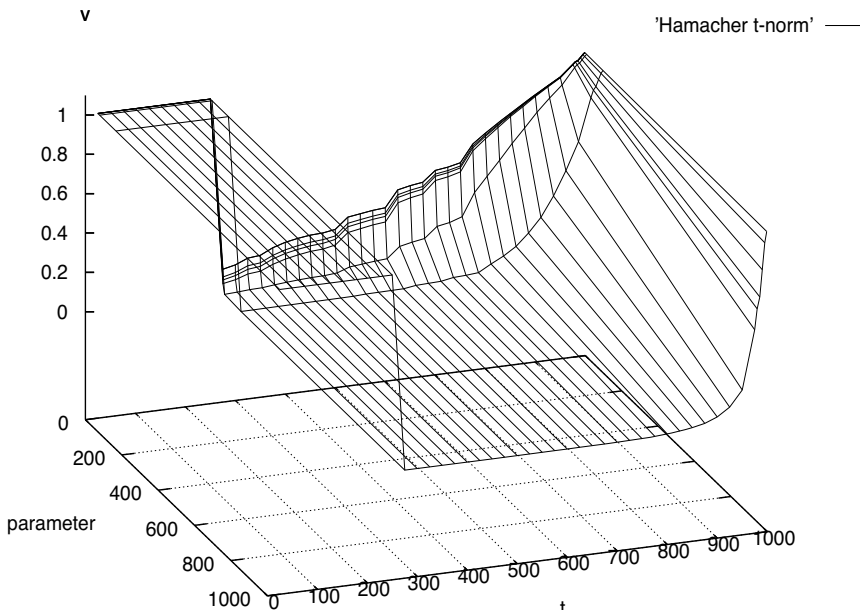

Fig. (16). Right random: $M$ using Hamacher.

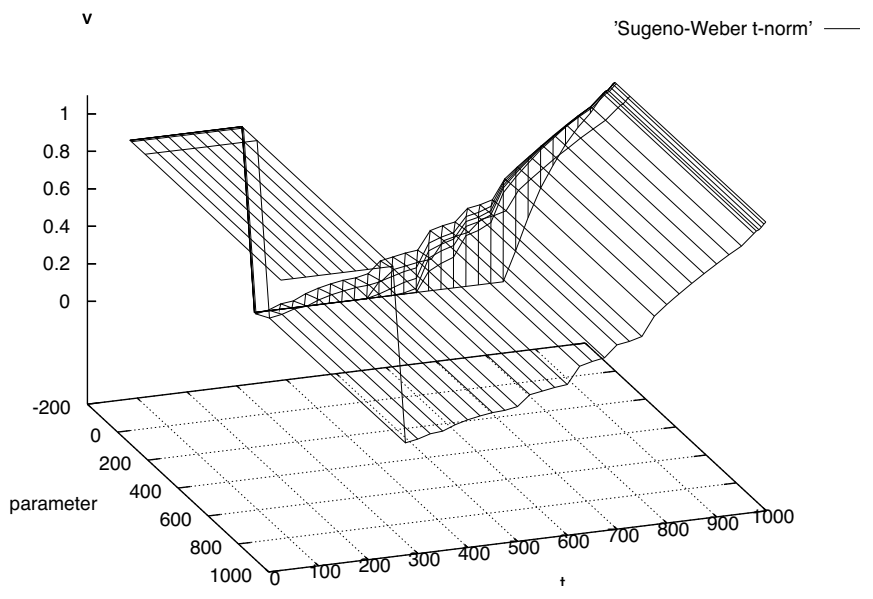

Fig. (17). Right random: M using Sugeno-Weber.

While they may prove to be very useful for other application scenarios (for instance non real-time systems) we have dropped them off our study due to their calculation complexity.

\section{APPLICATIONS OF THE FUZZY METRIC}

Obviously, to take advantage of the history of accesses evaluation, we need at least a history that is representative of the regular uses of the element. 


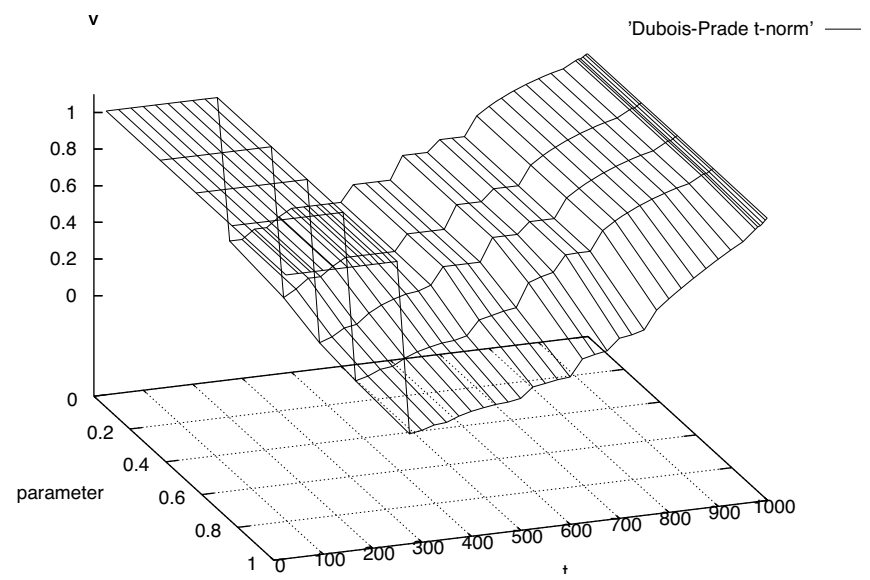

Fig. (18). Right random: M using Dubois-Prade.

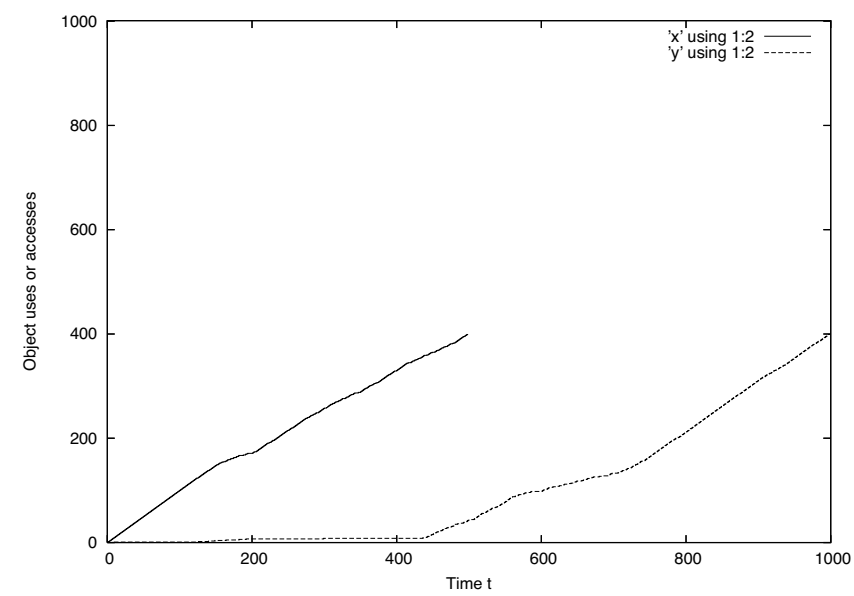

Fig. (19). Opposite histories.

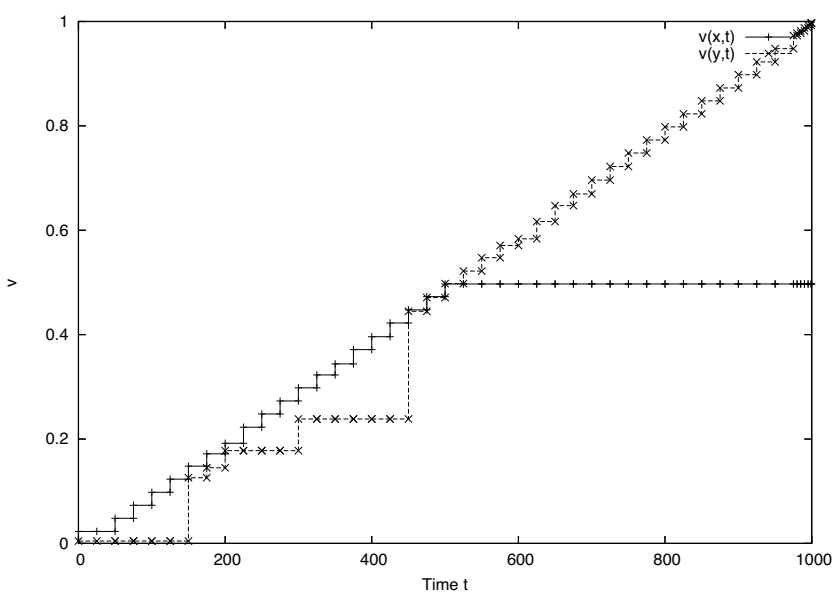

Fig. (20). Opposite histories: $v(x ; t)$ and $v(y ; t)$.

With the use of $M$ we can model situations where the value of $v$ is not high enough but the comparison of histories computed until a given moment $t$ tells that accesses are properly managed (or the other way around) when the metric considers that histories are close.

Naturally, the closer from $T$ the prediction is performed the more information we will have but also, in systems where erroneous assumptions require to undo previous operations (rollback in transactional database systems, for in- stance), we are likely to have less possibilities for turning back.

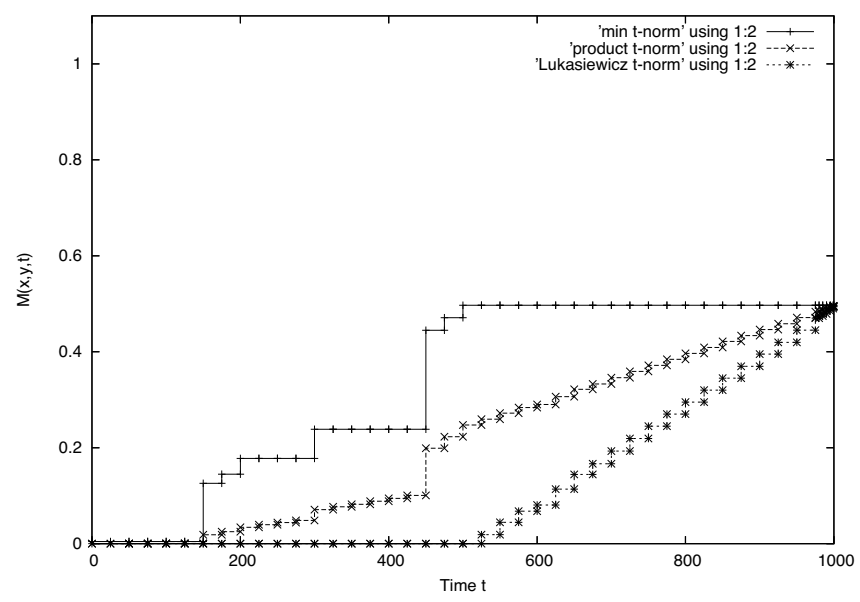

Fig. (21). Opposite histories: $M$ using minimum, product, Lukasiewicz.

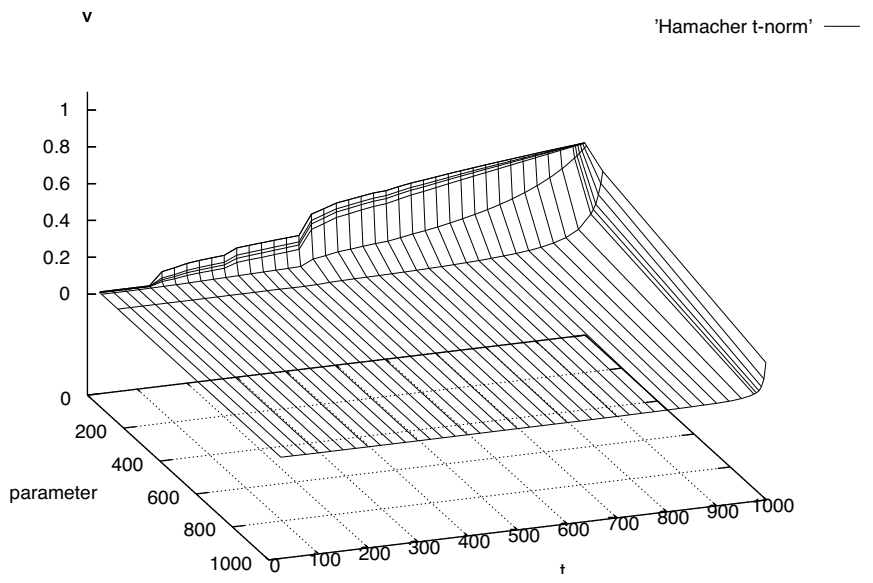

Fig. (22). Opposite histories: M using Hamacher.

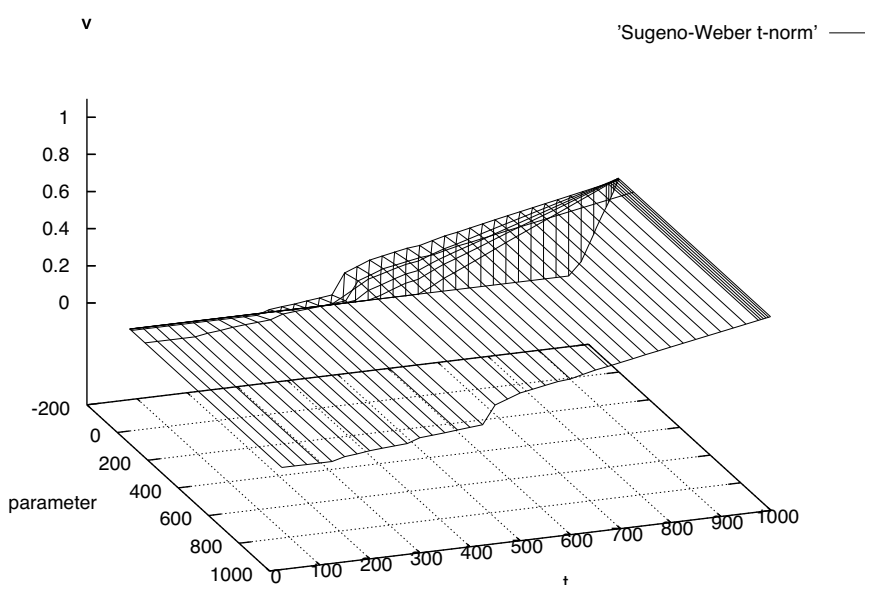

Fig. (23). Opposite histories: M using Sugeno-Weber.

\section{INTUITIONISTIC FUZZY METRIC EXTENSION}

For our next step, we will use the fact that the notion of an intuitionistic fuzzy metric space is a natural generalization of a fuzzy metric space. This fact allows us to adapt the idea of an intuitionistic fuzzy set presented by Atanassov in [2] so that we can measure the degree of nearness and the degree of 


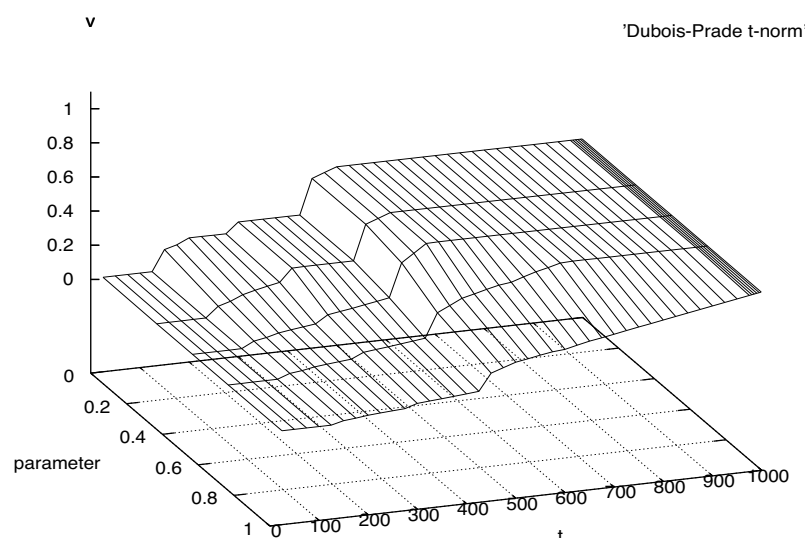

Fig. (24). Opposite histories: M using Dubois-Prade.

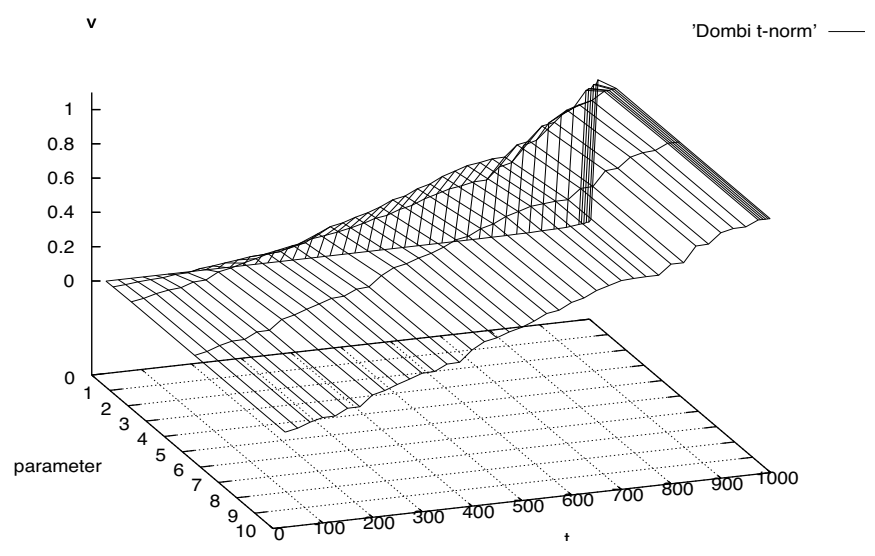

Fig. (25). Random: Dombi.

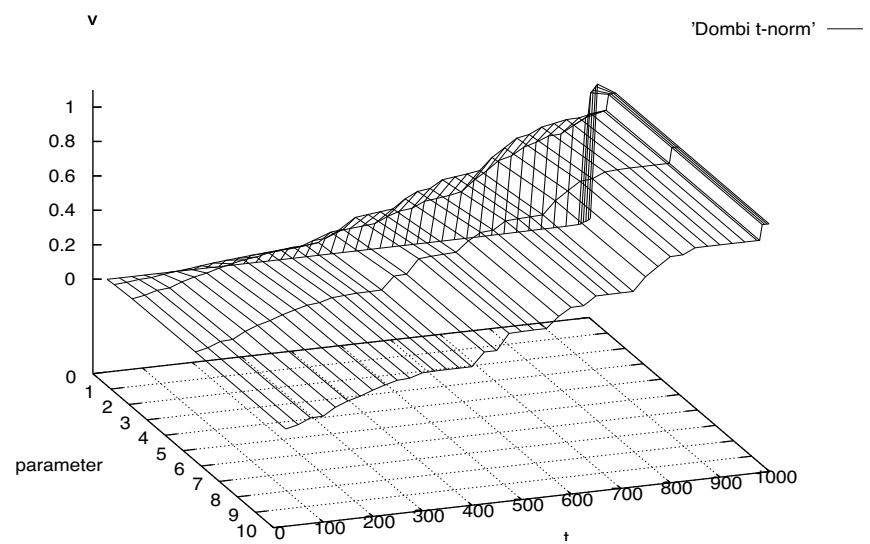

Fig. (26). Left-Random: Dombi.

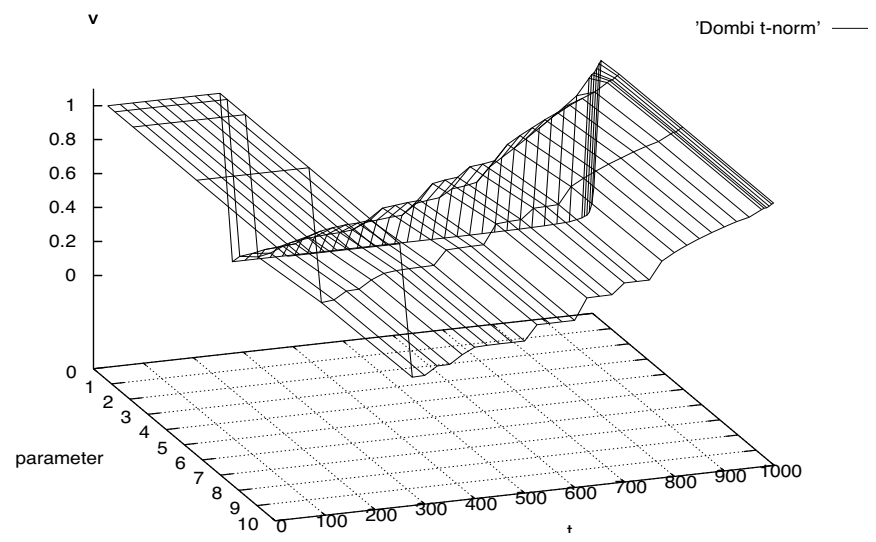

Fig. (27). Right-Random: Dombi.

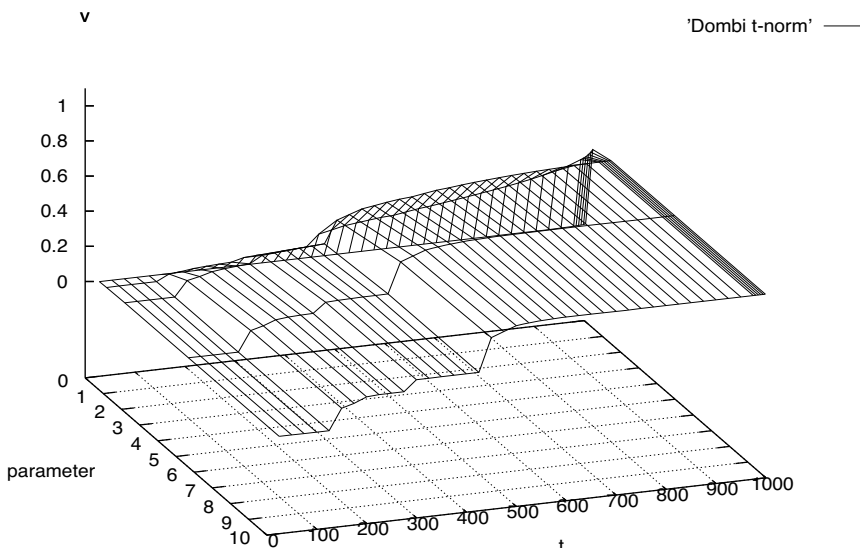

Fig. (28). Opposite histories: Dombi.

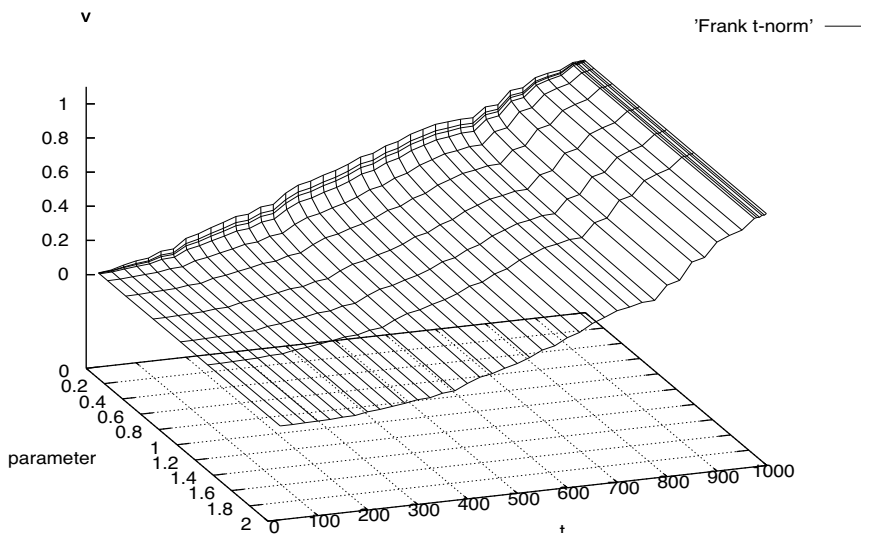

Fig. (29). Random: Frank.

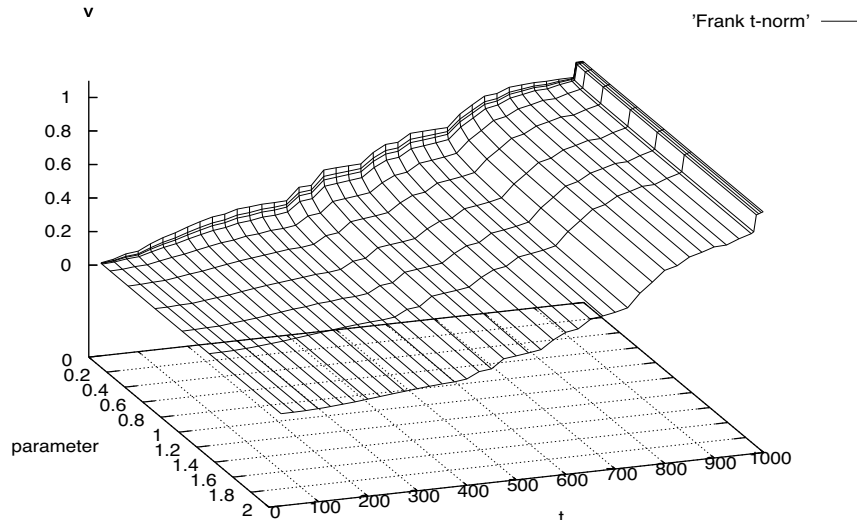

Fig. (30). Left-Random: Frank.

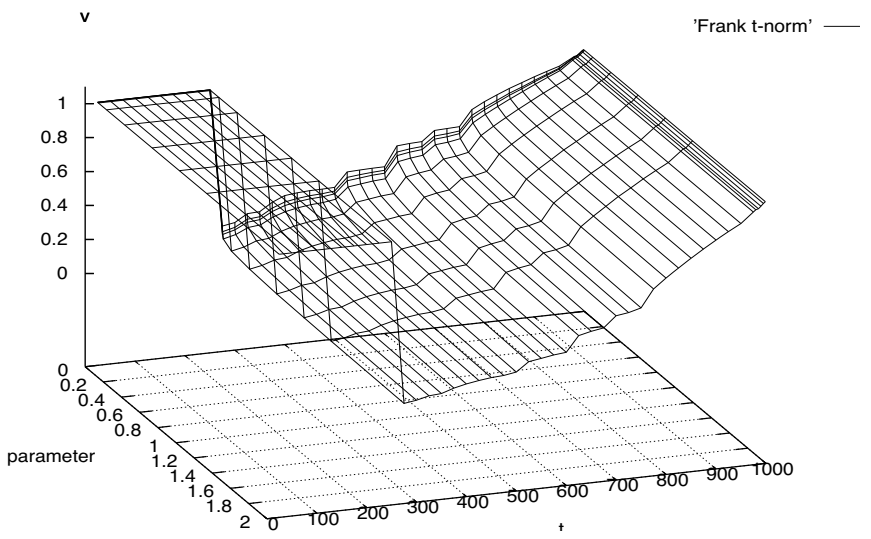

Fig. (31). Right-Random: Frank. 


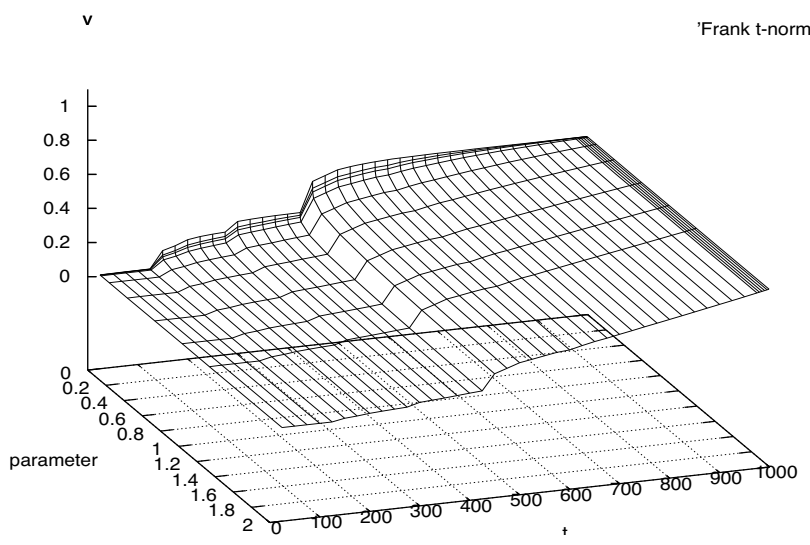

Fig. (32). Opposite histories: Frank.

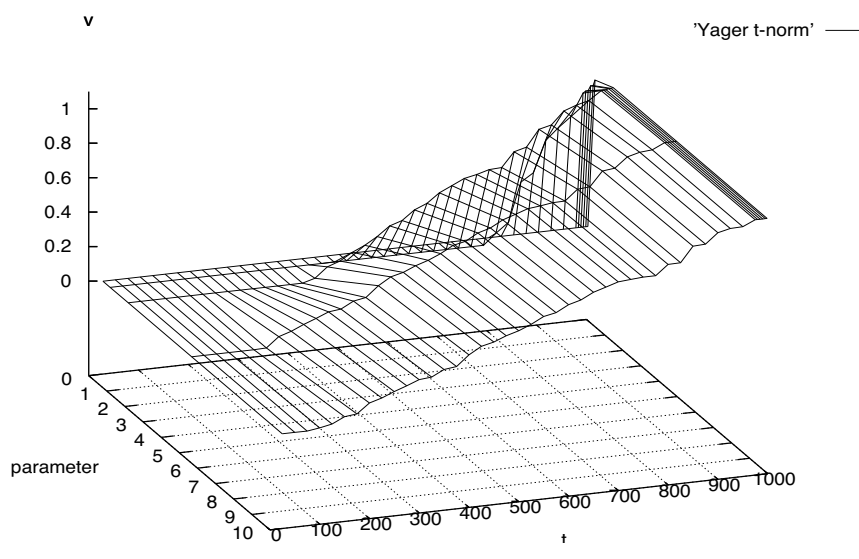

Fig. (33). Random: Yager.

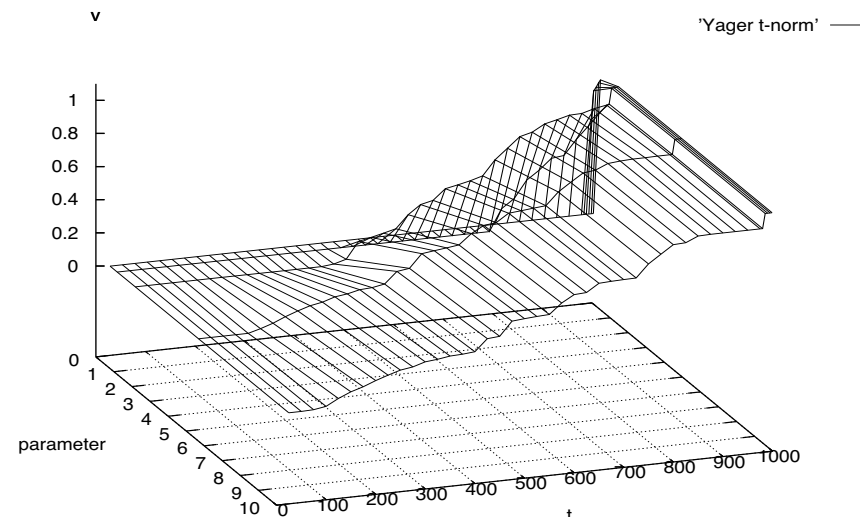

Fig. (34). Left-Random: Yager.

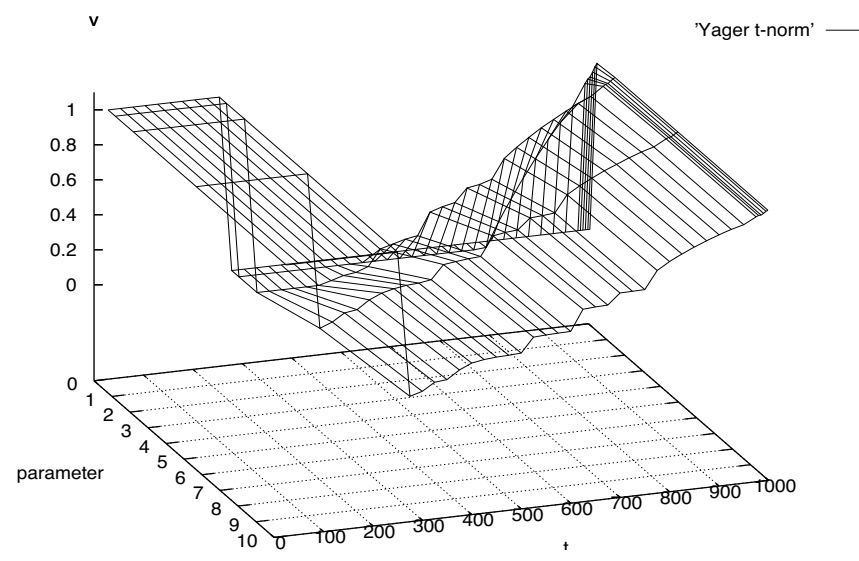

Fig. (35). Right-Random: Yager.

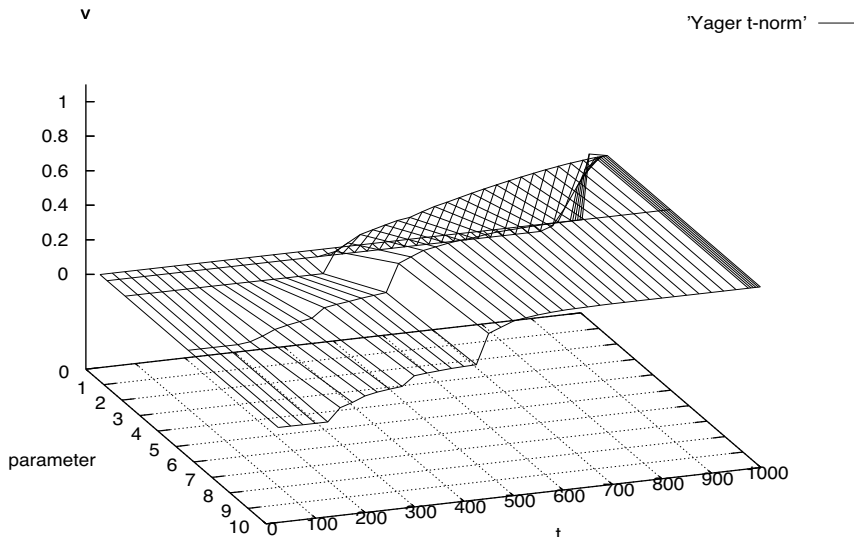

Fig. (36). Opposite histories: Yager.

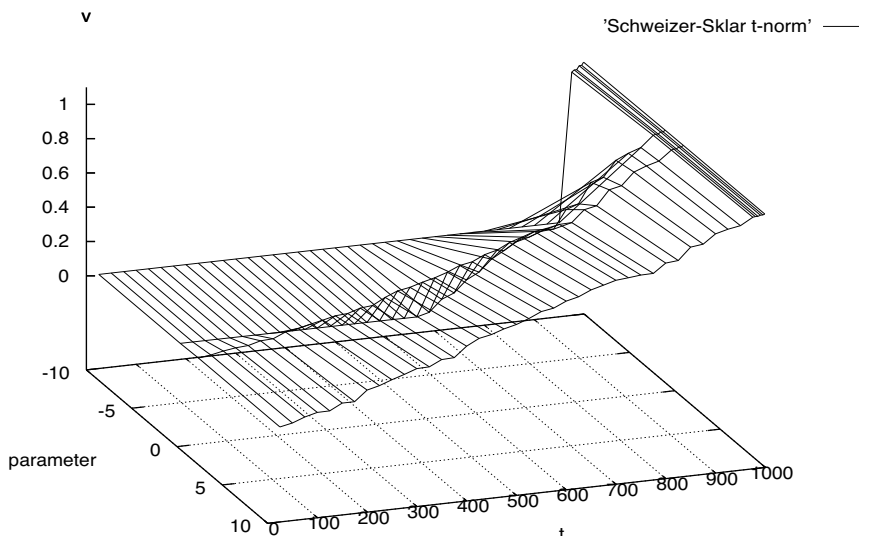

Fig. (37). Random: Schweizer-Sklar.

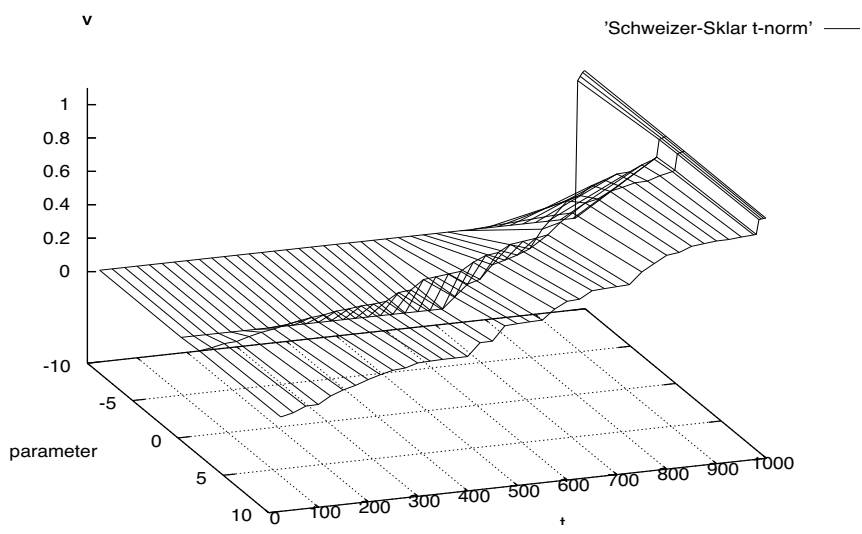

Fig. (38). Left-Random: Schweizer-Sklar.

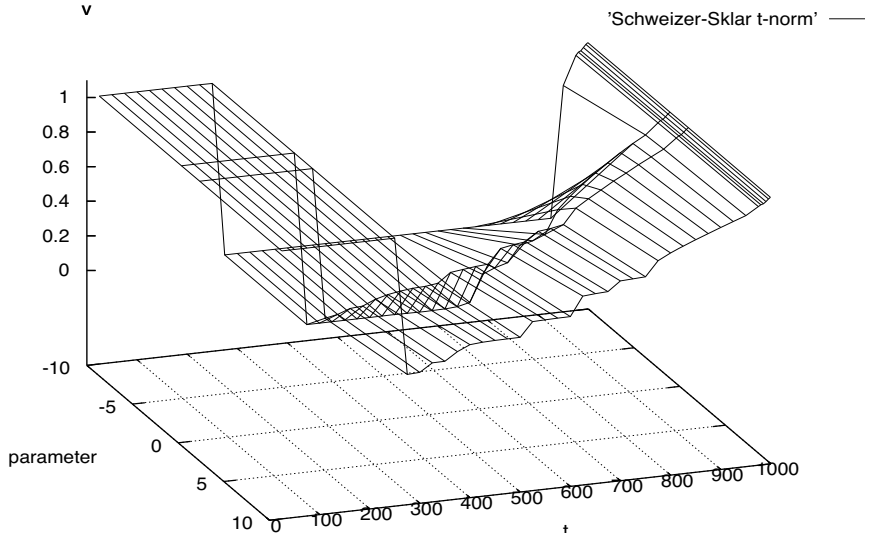

Fig. (39). Right-Random: Schweizer-Sklar. 


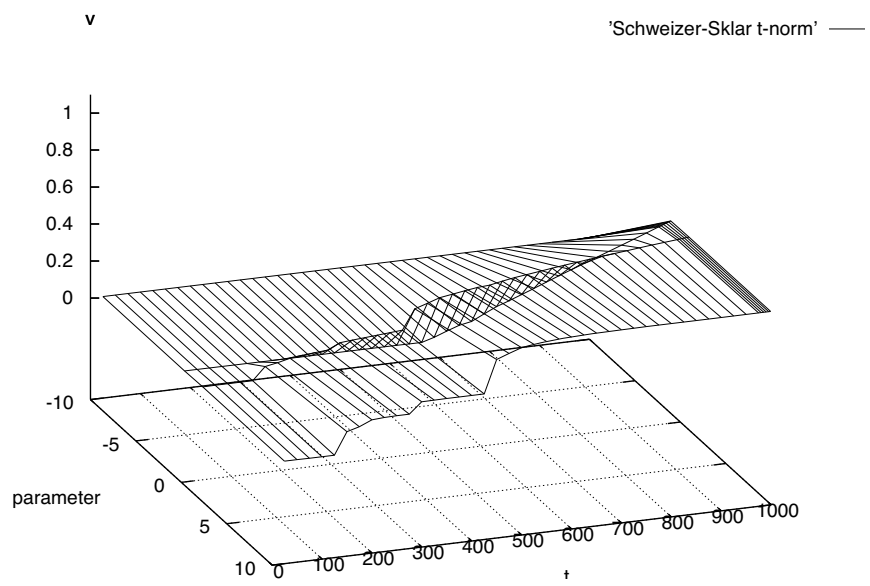

Fig. (40). Opposite histories: Schweizer-Sklar.

remoteness between two access histories. In our case, it is necessary to use the generalization defined by Alaca et al. in [11], a study that was later enriched by work of Romaguera and Tirado in [12].

\section{Definition 6 (Intuitionistic Fuzzy Metric Space)}

According to [11] and [12], an intuitionistic fuzzy metric space is a 5-tuple $(X, M, *, \diamond)$ such that $X$ is a non-empty set,, * a continuous t-norm, $\diamond$ is a continuous t-conorm and $M, N$ are sets in $X \times X \times[0, \infty)$ such that, for all $x, y, z \in X$ :

1) $M(x, y, t)+N(x, y, t) \leq 1$.

2) $M(x, y, 0)=0$.

3) $M(x, y, t)=M(y, x, t)$.

3) $M(x, y, t)=1$ for all $t>0 \Leftrightarrow x=y$.

4) $M(x, y, t)=M(y, x, t)$ for all $t>0$.

5) $M(x, y, t+s) \geq M(x, z, t) * M(z, y, s)$ for all $t, s \geq 0$.

6) $M(x, y\rfloor:,[0, \infty) \rightarrow[0,1]$ is left continuous.

7) $N(x, y, 0)=1$.

8) $N(x, y, t)=0$ for all $t>0 \Leftrightarrow x=y$.

9) $N(x, y, t)=N(y, x, t)$ for all $t>0$.

10) $N(x, y, t+s) \leq N(x, z, t) \diamond N(z, y, s)$ for all $t, s \geq 0$.

11) $N(x, y):,[0, \infty) \rightarrow[0,1]$ is left continuous.

In order to define a suitable function $N$ satisfying conditions 7) -11) of Definition 6, we need to find a new function $v$ ' that allows us to compare two elements in a given moment $t$ between 0 and $T$ and a set of t-conorms so that:

$$
N(x, y, t)=v^{\prime}(x, t) \diamond v^{\prime}(y, t) .
$$

While fuzzy sets introduce the concept of membership degree, intuitionistic fuzzy sets introduce the concept of nonmembership and uncertainty degrees (notice the first one of the properties in the definition of fuzzy metric space which also extends membership degrees to nearness degrees).

The following function $v^{\prime}$ is a suitable candidate to take advantage of the intuitionistic fuzzy spaces properties and to complement the representation of localized accesses histories:

We define $v^{\prime}: X \times[0, \infty) \rightarrow[0,1]$ by:

$$
\begin{gathered}
v^{\prime}(x, 0)=1, \\
v^{\prime}(x, t)=\prod_{j=1}^{k(x, t)} \frac{T-t_{j(x)}}{T}
\end{gathered}
$$

if $0<t \leq T$, and $v^{\prime}(x, t)=0$ if $t>T$.

Then we can obtain the following result (compare [9]).

Proposition 2: Let $v^{\prime}: X \times[0, \infty) \rightarrow[0,1]$ be any function such that for each $x \in X v^{\prime}(x$,$) is a left continuous non-$ increasing function (i.e., $v^{\prime}(x, t) \geq v^{\prime}(x, s)$ if $\left.t \leq s\right)$. $(X, 1-$ $\left.N, \diamond^{\prime}\right)$ is a fuzzy metric space where:

(a) $N(x, y, 0)=1$.

(b) $N(x, x, t)=0$ for all $t>0$ and for all $x \in X$.

(c) $N(x, y, t)=v(x, t) \diamond v(y, t)$ if $x \neq y$ for each $t>0$.

(d) $\diamond$ ' is the t-norm induced by $\diamond$, i.e., $a \diamond ' b=$

$1-[(1-a) \diamond(1-b)]$, for all $a, b \in[0,1]$.

Notice that our election of function $v$ ' satisfies Proposition 2, i.e., for each $x, v^{\prime}$ is left continuous and nonincreasing. Furthermore $M+N \leq 1$, so $(X, M, *, \diamond)$ is an intuitionistic fuzzy metric spaces, where $M$ was constructed before Proposition 1 .

\section{EVALUATED T-CONORMS}

Our source of continuous t-conorms is again Dubois and Prade [10]. After discarding too complex t-conorms, we have taken into account:

- Maximum: $\max (x, y):=\left\{\begin{array}{l}y \text { if } x \leq y \\ x \text { if } y<x\end{array}\right.$. It is the minimum dual.

- Product dual - Probabilistic sum:

$$
\prod^{\prime}(x, y):=x+y-x y
$$

- Lukasiewicz dual: $\mathrm{W}^{\prime}(x, y):=\min \{x+y, 1\}$.

We have also included figures showing the results for

- Hamacher':

$$
\frac{x+y+(\beta-1) x y}{1+\beta x y}
$$

where $\beta \geq-1$. It is the Hamacher family dual.

- Sugeno-Weber': $\min \{x+y+\lambda x y, 1\}$ where $\lambda \geq-1$.

It is the Sugeno-Weber family dual.

in order to see how their behaviour is very similar to the traditional t-norm duals.

The following figures illustrate the results obtained using the intuitionistic fuzzy metric for the same experiments that 
were performed for the t-norms. It will be worth revisiting Figs. $(1,7,13,19)$ while studying the next ones.

\section{Random Variations - Random}

For the random access cases (Figs. 41-44), it can be seen how the t-conorms ordering prevails and how fast $M$ values decrease due to $v^{\prime}(x, t)$ construction.

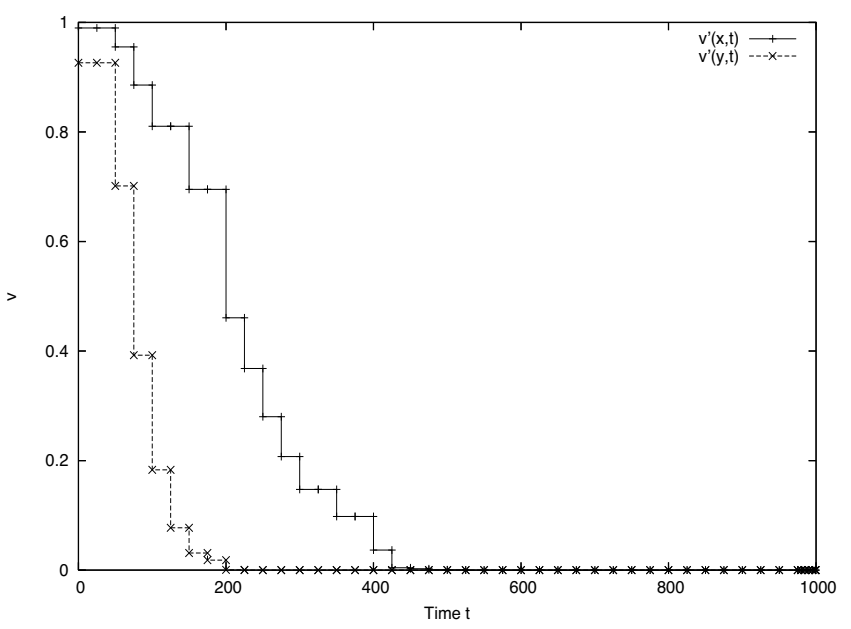

Fig. (41). Random: v'(x;t) and v'(y;t).

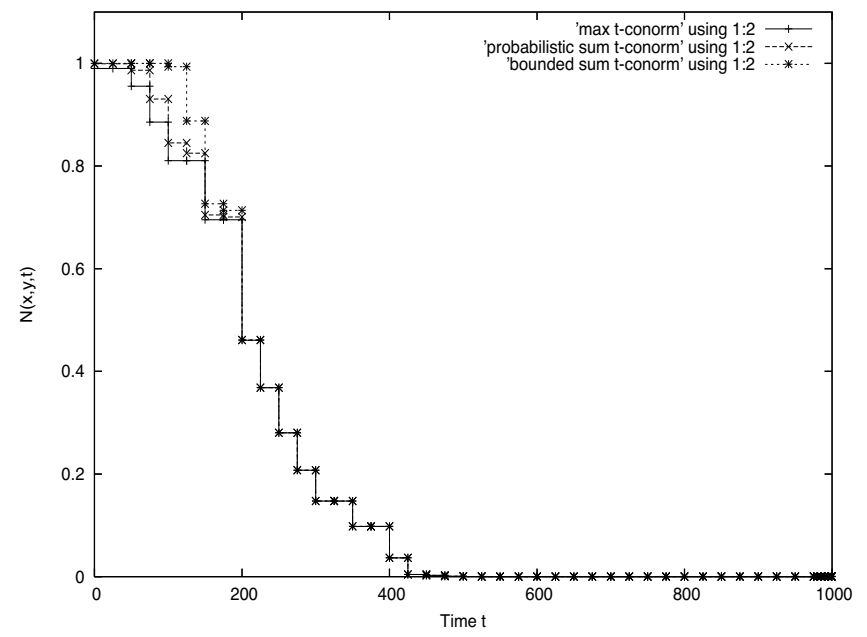

Fig. (42). Random: $N(x ; y ; t)$.

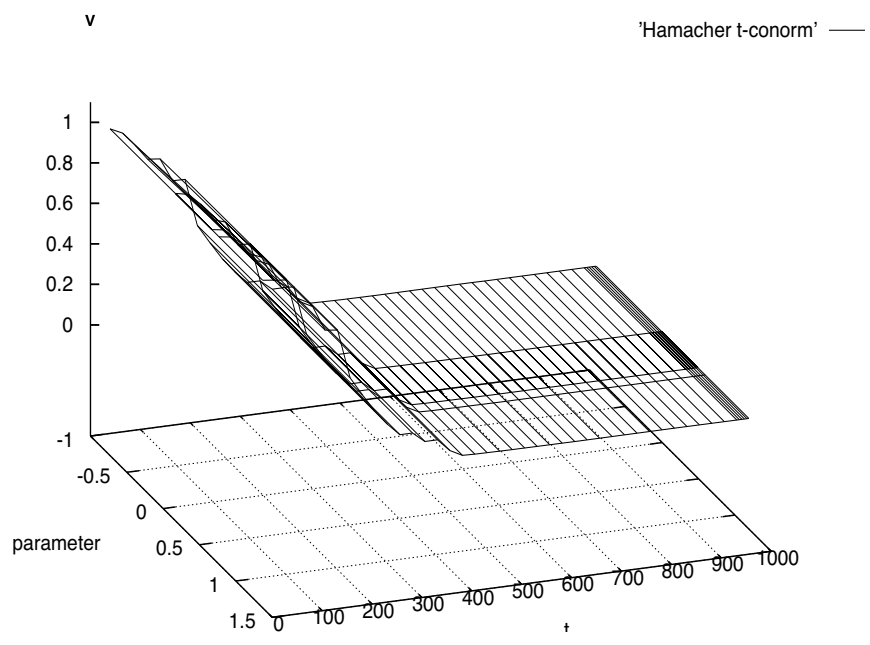

Fig. (43). Random: N using Hamacher'.

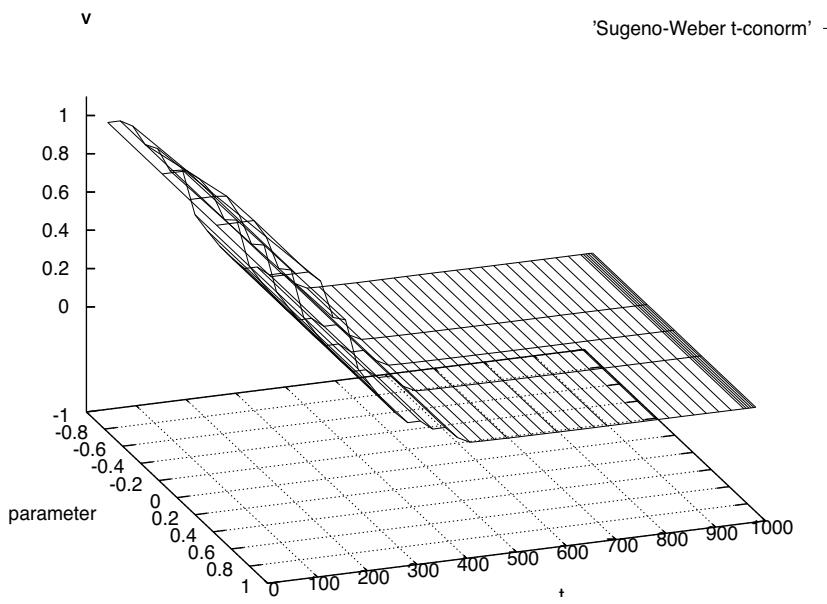

Fig. (44). Random: N using Sugeno-Weber'.

\section{Left-Random Variations - Left Random}

The experiments for left-random accesses (Figs. 45-48), display how $N$ identifies the differences between element histories concentrated near to the starting point. From a given moment on, both histories show an identical access pattern and $N$ returns very close to 0 values. This characteristic is reinforced by the fact that $k(x)$ and $k(y)$ are big enough for the product to tend to 0 . As soon as we come across a situation like this, we must consider that histories distance is measured by $v^{\prime}$ better than using $N$.

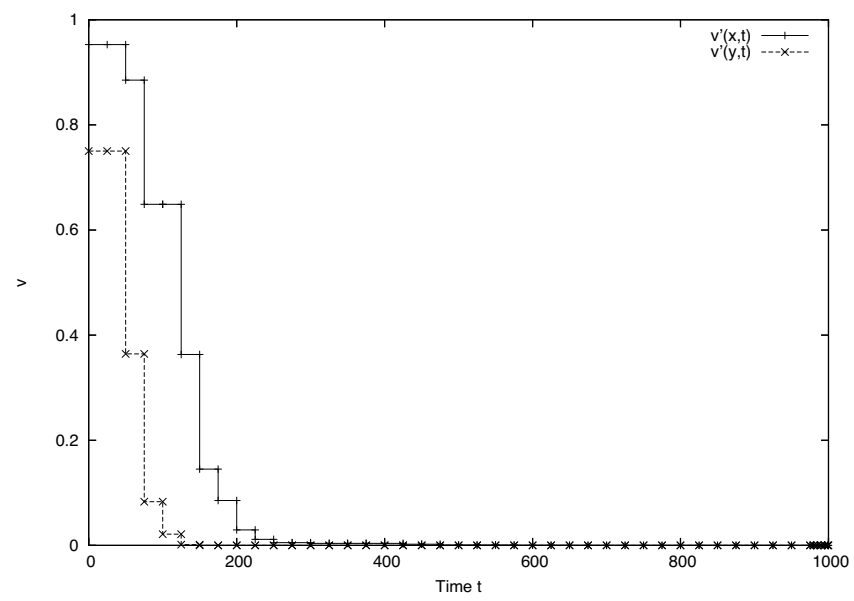

Fig. (45). Left-Random: $v^{\prime}(x ; t)$ and $v^{\prime}(y ; t)$.

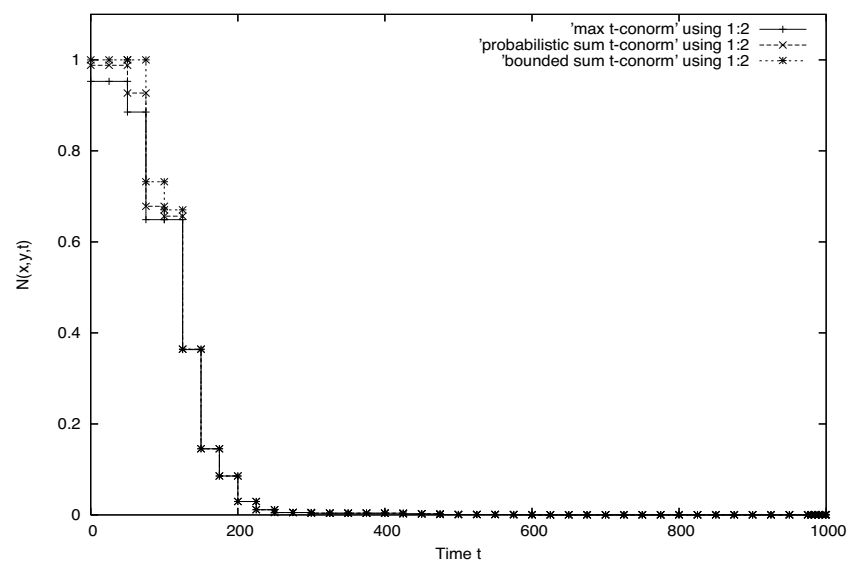

Fig. (46). Left-Random: $\mathrm{N}(\mathrm{x} ; \mathrm{y} ; \mathrm{t})$. 


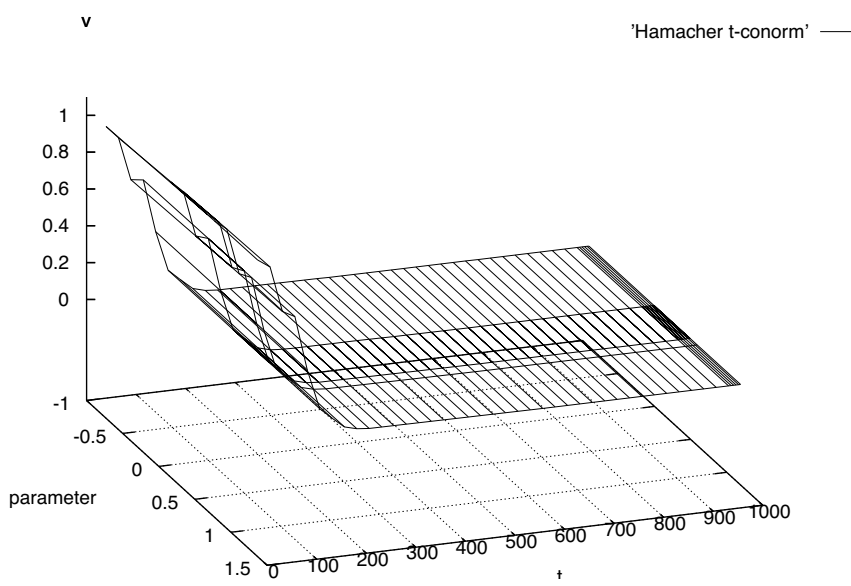

Fig. (47). Left-Random: $\mathrm{N}$ using Hamacher'.

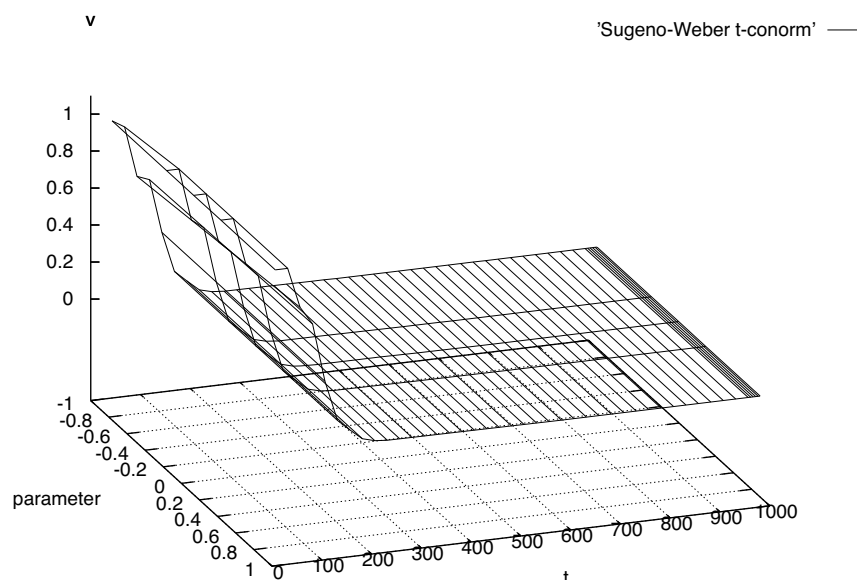

Fig. (48). Left-Random: N using Sugeno-Weber'.

\section{Right-Random Variations - Right Random}

For the right-random experiments histories (Figs. 49-52), divergence starts at the moment $T / 4$. In that same moment $N$ starts displaying differences until it turns 0 again due to the amount of accesses already performed (similar to the leftrandom accesses case).

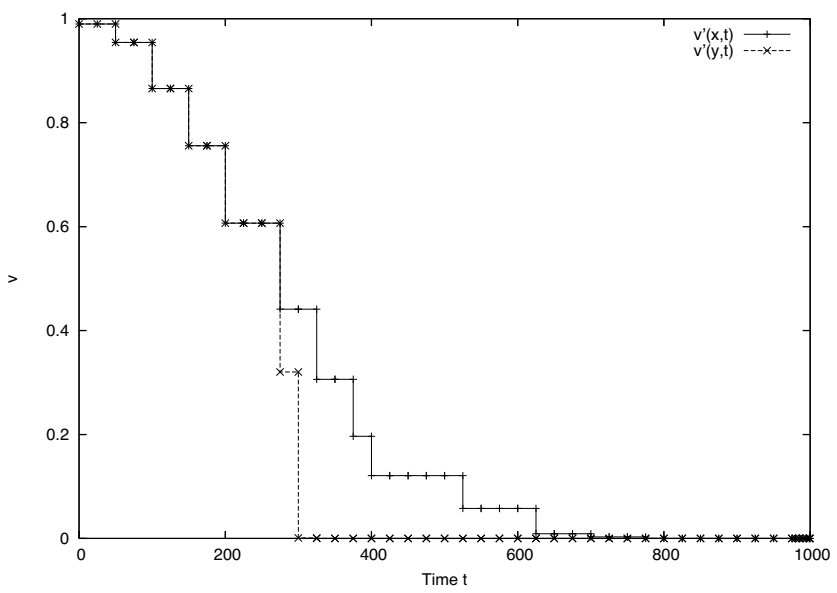

Fig. (49). Right random: $v^{\prime}(x ; t)$ and $v^{\prime}(y ; t)$.

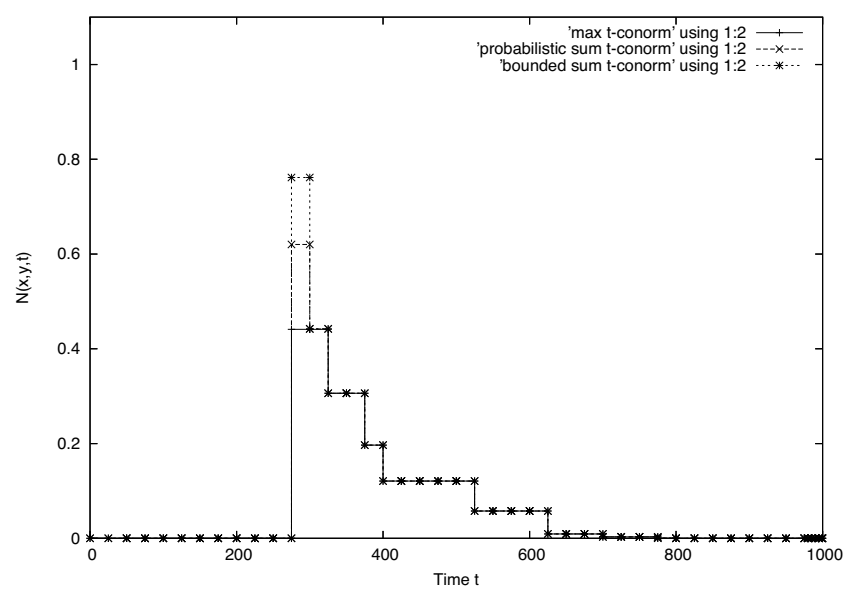

Fig. (50). Right random: $N(x ; y ; t)$.

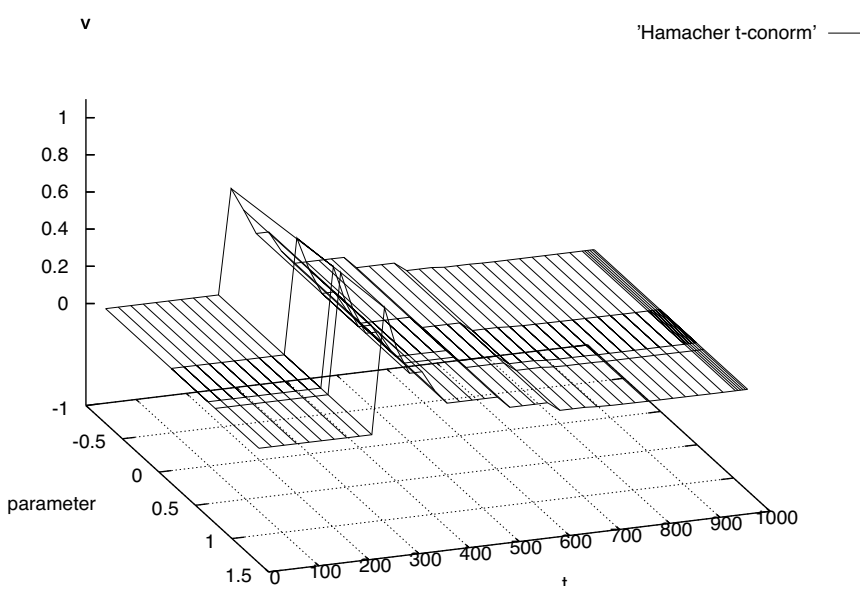

Fig. (51). Right random: $N$ using Hamacher'.

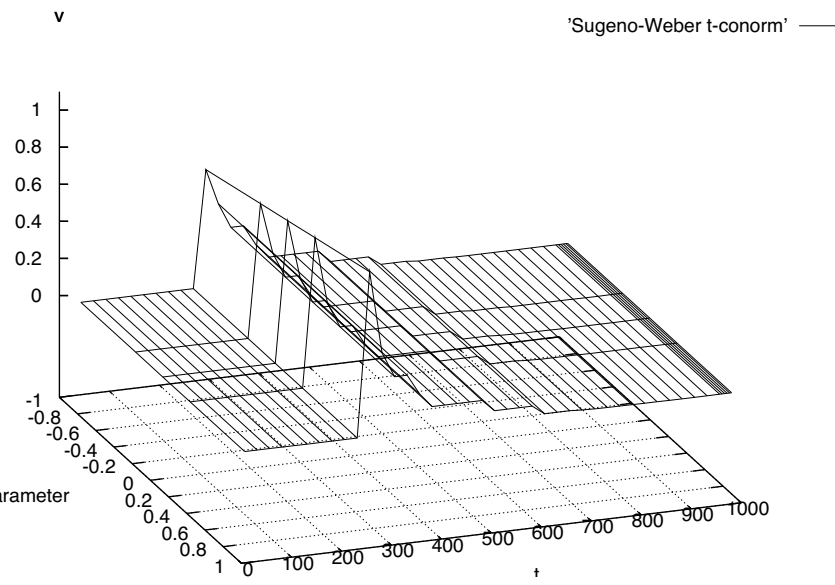

Fig. (52). Right random: N using Sugeno-Weber'.

\section{Two Independent Histories - Opposite Histories}

When we compare the opposite histories experiments (Figs. 53-56), with the ones from the right-random accesses, $N$ decrement for the opposite histories case is softer than for the right-random case, as one might have expected because differences already appear far from the end of the histories measuring time end. 
As in the rest of the cases, history differences towards the end of the history are not compared as explicitly as the comparisons performed towards the beginning of the history due to the amount of already performed accesses.

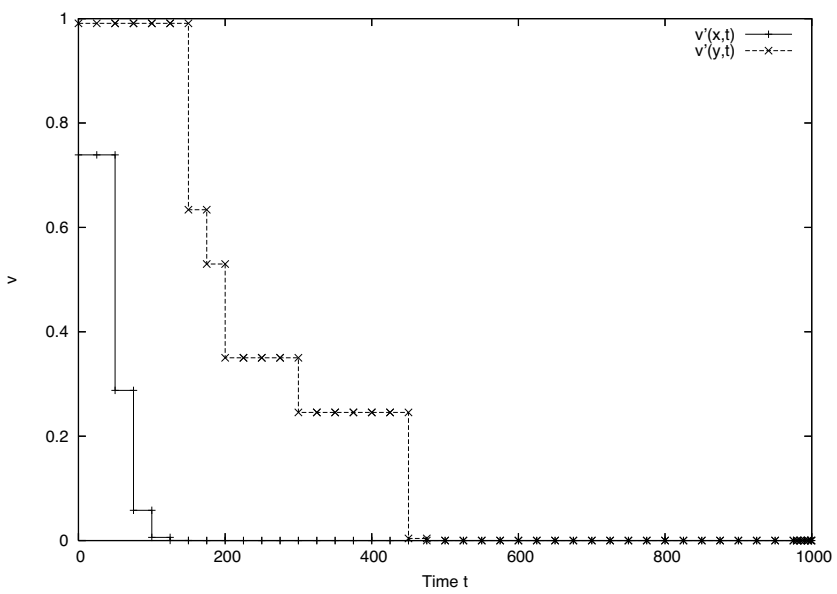

Fig. (53). Opposite histories: v'(x;t) and $v^{\prime}(y ; t)$.

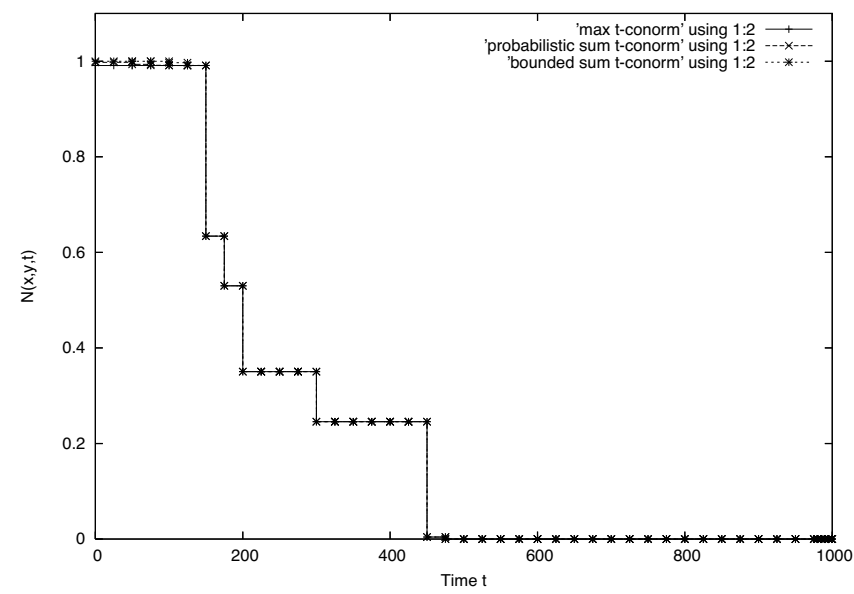

Fig. (54). Opposite histories: $\mathrm{N}(\mathrm{x} ; \mathrm{y} ; \mathrm{t})$.

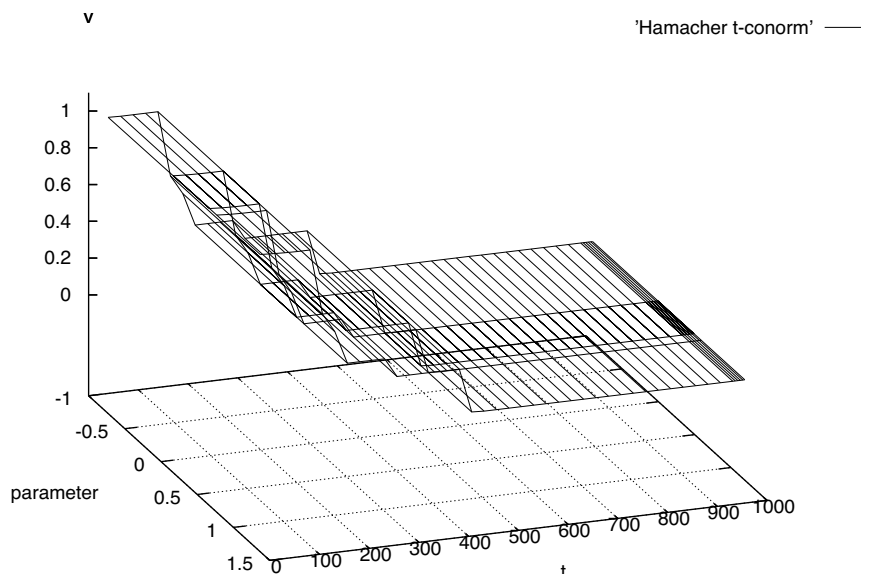

Fig. (55). Opposite histories: N using Hamacher'.

\section{INTUITIONISTIC FUZZY METRIC APPLICATIONS}

The results for the experiments obtained with $v$ ' and the t-conorms are shown in the following figures on top of the ones obtained with $v$ and the t-norms to show the intuitionis- tic fuzzy metric behaviour (applying the correction factor $M / 2$ and $N / 2$ in order to fulfill all the intuitionistic fuzzy metric properties).

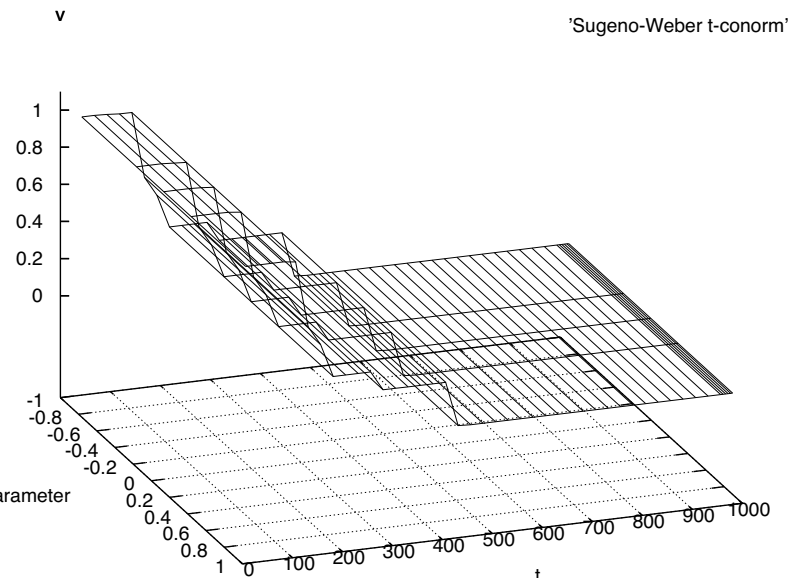

Fig. (56). Opposite histories: N using Sugeno-Weber'.

Fig. (57-60) show the results of $M$ and $N$ obtained for the previous 4 scenarios. We have only shown the results for traditional t-norms because they act as boundaries (begin the minimum the upper bound of all of them) for the considered families.

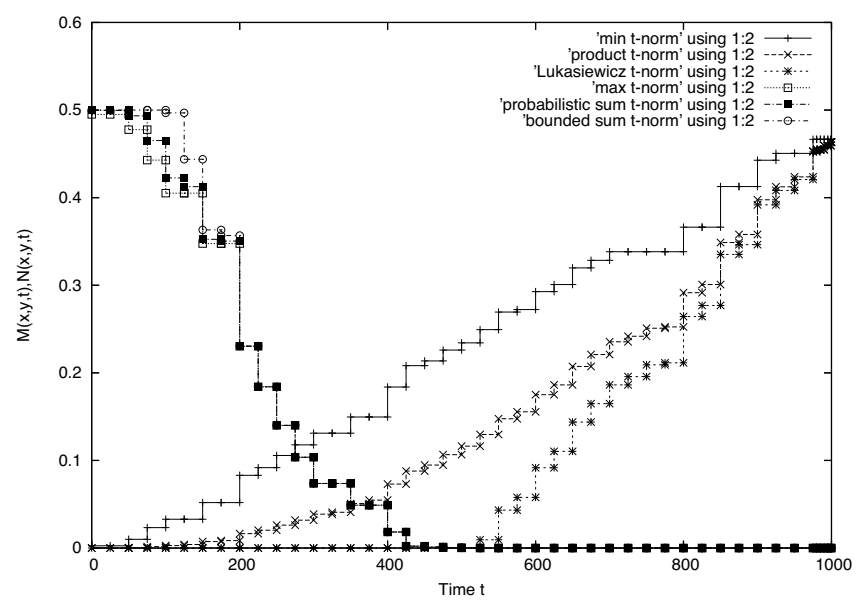

Fig. (57). Random: $M$ and N.

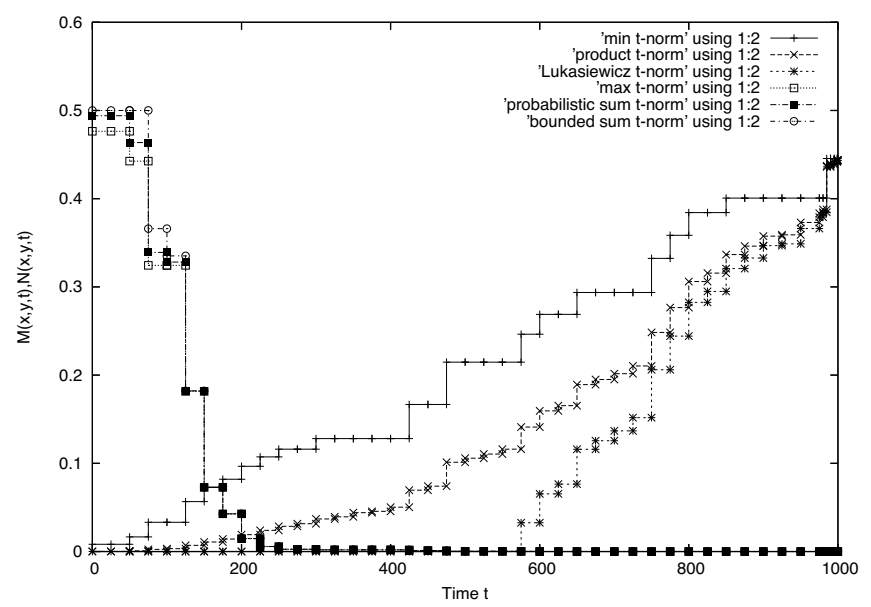

Fig. (58). Left-Random: $M$ and $N$. 


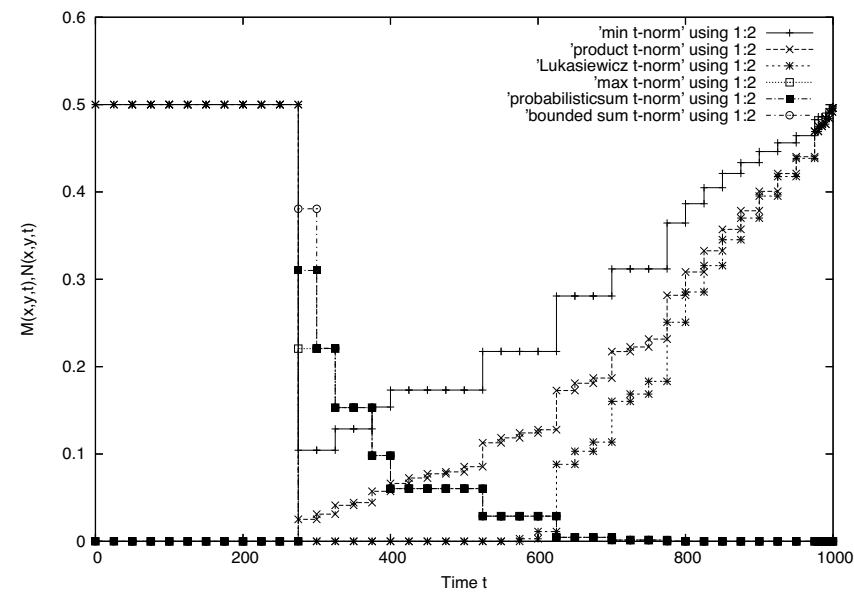

Fig. (59). Right-Random: $M$ and $N$.

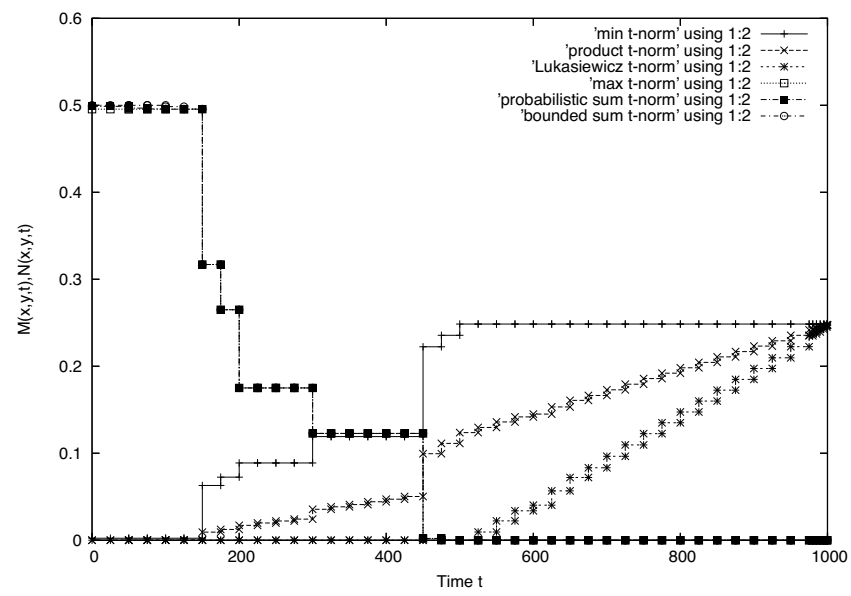

Fig. (60). Opposite histories: $M$ and $N$.

Families are then useful if we need a finer tuning or if our system behaves adaptively and the family parameter is able to introduce subtle improvements in the predictions. This also confirms that for a general scenario there is no such thing as an optimal parameter.

Due to the fact that $M$ represents the nearness degree and $N$ represents the remoteness degree, the intuitionistic fuzzy metric allows us to use two thresholds: one for "goodness" and another one for "badness".

For each scenario the intersection between a function $M$ defined by a t-norm and a function $N$ defined by a t-conorm (not necessarily the dual one) expresses the moment when a history is not "distant" anymore and becomes "close".

Notice that allowing a degree of uncertainty makes $N$ useful. Otherwise it would not offer more information than the one we already had with $M$.

Initially, if the uncertainty degree is "reasonable":

- If $M(x, y, t)>N(x, y, t)$ then we can say that both elements belong to the same class.

- If $N(x, y, t)>M(x, y, t)$ then we can say that they belong to different classes.
- If $N(x, y, t)=M(x, y, t)$ then we cannot confirm anything about class membership solely with the result of the intuitionistic fuzzy metric.

For the latter case, we can always choose to decide optimistically (consider them from the same class) or pessimistically (consider them from different classes).

\section{CONCLUSIONS AND FUTURE WORK}

We have shown that the optimization of accesses in systems based on locality can be achieved using a mathematical framework based on intuitionistic fuzzy metric spaces. In this work, we present experimental results representing best, average and worst cases for a variety of elections in the fuzzy constructions we can build. Combination of the different t-norm and t-conorms allows us to model the general case of accesses locality.

Our next steps will consist on the application of these fuzzy metric techniques in order to find optimal thresholds for classification into classes and decisions regarding the "goodness" and "badness" of those.

One possibility is to make use of the multiple variations we can get by combining different t-norms and t-conorms. Another one would be to change the definition of $v$ and $v$ while maintaining their definition in the scope of the intuitionistic fuzzy metric. Special care has to be taken for the election of $v$ ': Our current election is built using a product and its results decrease very abruptly.

In general, our results show that traditional continuous tnorms are the constructs we have to base upon in order to build the metric. We find especially outstanding the results obtained for the minimum and Lukasiewicz t-norms which are fast to compute and discriminate history results better than the rest.

T-norm families rather than introducing complexity, introduce a very interesting possibility for finer tuning. This makes our model extremely configurable and suitable for a range of possible future applications much greater than our initial study [7] which already proved useful for replicated database systems.

\section{ACKNOWLEDGEMENT}

Salvador Romaguera's work partially supported by Ministerio de Educación y Ciencia and FEDER grant MTM2006-14925-C02-01.

\section{REFERENCES}

[1] L. A. Zadeh, "Fuzzy sets", Inform. Control, vol. 8, pp. 338-353, 1965.

[2] K. Atannasov, "Intuitionistic fuzzy sets”, Fuzzy Set Syst., vol. 20 pp. 87-96, 1986 .

[3] I. Kramosil and J. Michalek, "Fuzzy metrics and statistical metric spaces", Kybernetika, vol. 11, pp. 326-334, 1975.

[4] A. George and P. Veeramani, "On some results in fuzzy metric spaces”, Fuzzy Set Syst., vol. 64, pp. 395-399, 1994.

[5] O. Kaleva and K. Seikkala, "On fuzzy metric spaces", Fuzzy Set Syst., vol. 12, pp. 215-229, 1984.

[6] G. Artico and P. Moresco, "On fuzzy metrizability", J. Math. Anal. Appl., vol. 107, pp. 114-147, 1985. 
[7] F. Castro-Company, S. Romaguera, J. M. Sánchez-Álvarez, and P. Tirado, "A quasi-metric lattice approach for access prediction in replicated database systems", in International e-Conference on Computer Science, Proc. American Institute of Physics (to appear), 2007

[8] K. Menger, "Statistical metrics", in Proc. National Acad. Sci. of the United States of America, vol. 48, pp. 535-7, 1942.

[9] F. Castro-Company and S. Romaguera, "Intuitionistic fuzzy metric spaces applications for access history predictions", work in progress, 2008.
[10] D. Dubois and H. "Prade, Fundamentals of Fuzzy Sets", The Handbooks of Fuzzy Sets Series. Kluwer Academic, 2000

[11] C. Alaca, D. Turkoglu, and C. Yildiz, "Fixed points in intuitionistic fuzzy metric spaces", Chaos, Soliton Fract., vol. 29, pp. 1073$1078,2006$.

[12] S. Romaguera and P. Tirado, "On fixed point theorems in intuitionistic fuzzy metric spaces", Internat. J. Nonlinear Sci. Numer. Simul., vol. 8, pp. 233-238, 2007.

(C) Castro-Company and Romaguera; Licensee Bentham Open.

This is an open access article distributed under the terms of the Creative Commons Attribution License (http://creativecommons.org/licenses/by/2.5/), which permits unrestrictive use, distribution, and reproduction in any medium, provided the original work is properly cited. 